\title{
SN 1991 bg - A type IA supernova with a difference
}

\section{Citation}

Leibundgut, Bruno, Robert P. Kirshner, Mark M. Phillips, Lisa A. Wells, N. B. Suntzeff, Mario Hamuy, R. A. Schommer, et al. 1993. "SN 1991 bg - A Type IA Supernova with a Difference." The Astronomical Journal 105 (January): 301. https://doi.org/10.1086/116427.

\section{Permanent link}

http://nrs.harvard.edu/urn-3:HUL.InstRepos:41417333

\section{Terms of Use}

This article was downloaded from Harvard University's DASH repository, and is made available under the terms and conditions applicable to Other Posted Material, as set forth at http:// nrs.harvard.edu/urn-3:HUL.InstRepos:dash.current.terms-of-use\#LAA

\section{Share Your Story}

The Harvard community has made this article openly available.

Please share how this access benefits you. Submit a story.

\section{Accessibility}


SN 1991bg: A TYPE Ia SUPERNOVA WITH A DIFFERENCE ${ }^{1}$

\title{
BRUNO LEIBUNDGUT ${ }^{2}$ AND ROBERT P. KIRSHNER
}

Harvard-Smithsonian Center for Astrophysics, 60 Garden Street, Cambridge, Massachusetts 02138

Mark M. Phillips, Lisa A. Wells, N. B. Suntzeff, Mario Hamuy, R. A. Schommer,

A. R. Walker, L. Gonzalez, P. Ugarte, R. E. Williams, and G. Williger

Cerro Tololo Inter-American Observatory, ${ }^{3}$ Casilla 603, La Serena, Chile

\section{Mercedes Gomez, Ronald Marzke, Brian P. Schmidt, and Barbara Whitney}

Harvard-Smithsonian Center for Astrophysics, 60 Garden Street, Cambridge, Massachusetts 02138

Nelson Caldwell ${ }^{4}$ and J. Peters

F. L. Whipple Observatory, Box 97, Amado, Arizona 85645

F. H. Chaffee and Craig B. Foltz

Multiple Mirror Telescope Observatory, Tucson, Arizona 85721

D. REHNER ${ }^{4,5}$ AND L. Siciliano ${ }^{4}$

Space Telescope Science Institute, 3700 San Martin Drive, Baltimore, Maryland 21218

T. G. BARNES ${ }^{4}$

The University of Texas at Austin, McDonald Observatory, Austin, Texas 78712

K.-P. CHENG $^{4}$

NASA/Goddard Space Flight Center, Code 684, Greenbelt, Maryland 20771

\section{P. M. N. HINTZEN ${ }^{4}$}

University of Nevada, Department of Physics, Las Vegas, Nevada 89154

$$
\text { Y.-C. } \mathrm{KIM}^{4}
$$

Yale University, Department of Astronomy, P.O. 6666, New Haven, Connecticut 06511

JOSE MAZA ${ }^{4}$

Universidad de Chile, Observatorio Astronómico Nacional, Casilla 36-D, Santiago, Chile

\section{J. WM. PARKeR ${ }^{4}$}

APAS/CASA, University of Colorado, Campus Box 391, Boulder, Colorado 80309

A. C. PORTER

National Optical Astronomy Observatory, P.O. Box 26732, Tucson, Arizona 85726

P. C. SCHMIDTKE ${ }^{4}$

Arizona State University, Department of Physics and Astronomy, Tempe, Arizona 85287

\section{GEORGE SONNEBORN}

Laboratory for Astronomy and Solar Physics, NASA/Goddard Space Flight Center, Code 681, Greenbelt, Maryland 20771 Received 18 June 1992; revised 14 August 1992

\begin{abstract}
We present 13 spectra and 31 photometric observations covering the first 150 days of SN 1991bg in NGC 4374 (M 84). Although SN 1991bg was a type Ia supernova displaying the characteristic Si II absorption at $6150 \AA$ near maximum and the $\mathrm{Fe}$ emission lines at late phases, it varied from the well-defined norm for SNe Ia in several important respects. The peculiarities include faster declines in the $B$ and $V$ light curves after maximum, a distinct color evolution, a very red $B-V$ color near maximum, relatively faint peak luminosity, a distinct spectral evolution, and a short peak phase. The narrow peak of the luminosity and the rapid declines of the light curves suggest a smaller mass in the
\end{abstract}


ejecta and larger energy losses than for most SNe Ia. The unusually red color at maximum is not a result of normal extinction, since SN 1991bg was as blue as other SNe Ia at late times and no narrow interstellar lines are observed in the spectra. The faint absolute magnitude of SN $1991 \mathrm{bg}$ is established beyond doubt by comparison with SN 1957B, another type Ia supernova in the same galaxy, which was $\sim 2.5$ magnitudes brighter than SN 1991bg. The spectral evolution reveals minor differences near maximum compared to other well-observed SNe Ia, mainly in relative line strengths. At later phases several wavelength regions display discrepancies when compared to spectra of normal SNe Ia. Although other SNe Ia, such as SN 1986G and SN 1939B, have light curves with fast decline rates, SN $1991 \mathrm{bg}$ is unique, with deviations in both light curves and spectra. In particular SN 1991bg is the only SN Ia observed to date with a distinct spectrum at $\sim 40$ days past maximum. Although $\mathrm{SN} 1991 \mathrm{bg}$ is an extreme case, with unusual photometric and spectroscopic properties, we believe it can be understood in the context of exploding white dwarf models, and is properly grouped with type Ia. SN 1991bg demonstrates the need for detailed observations of SNe Ia as part of their use as standard candles for cosmology. While there is a well-defined prototype with homogeneous properties, unusual cases like SN 1991 bg must be identified and separated to avoid misleading results.

\section{INTRODUCTION}

The discovery of a number of bright supernovae of type Ia ( $\mathrm{SNe}$ Ia) in recent years has focused unprecedented attention on these events (Phillips et al. 1987, 1992; Frogel et al. 1987; Leibundgut 1988; Filippenko 1989; Leibundgut et al. 1991a,b; Hamuy et al. 1991; Filippenko et al. 1992a,b; Ruiz-Lapuente 1992; Ruiz-Lapuente et al. 1992; Wells et al. 1992). The homogeneity of the spectral and photometric appearance of this class of supernovae is often taken to indicate a single explosion mechanism and has independently generated interest in the possible uses of $\mathrm{SNe}$ Ia as distance indicators. Such a hypothesis must, however, be checked with each well-observed supernova to test the assumptions, and to explore intrinsic differences among individual events. Deviations from the norm, like distinct light curves or variations in spectral evolution, are important clues to the nature of SN Ia explosions, and provide valuable information on possible variations on a general theme.

Supernovae are divided into two main classes on the basis of the presence (type II) or absence (type I) of hydrogen lines in their maximum light spectrum (Minkowski 1941, 1964; Kirshner et al. 1973; Harkness \& Wheeler 1990). Separations into more classes (Zwicky 1962, 1965) have been abandoned, since only one object of each peculiar type was known. A potential pitfall of any spectral classification system is that it might be based on insignificant details of surface conditions and might not provide a physically based separation of fundamentally different events. On the other hand, a classification scheme should

\footnotetext{
${ }^{1}$ Work based partly on observations at the Multiple Mirror Telescope, a joint facility of the Smithsonian Astrophysical Observatory and the University of Arizona.

${ }^{2}$ Visiting Astronomer, Kitt Peak National Observatory, National Optical Astronomy Observatories.

${ }^{3}$ Operated by the Association of Universities for Research in Astronomy, Inc. (AURA) under cooperative agreement with the National Science Foundation.

${ }^{4}$ Visiting Astronomer, Cerro Tololo Inter-American Observatory, National Optical Astronomy Observatories.

${ }^{5}$ Astronomy Programs, Computer Sciences Corporation.
}

provide an easy tool to distinguish different types on observational grounds-so spectra near maximum are practical. This contradiction is not readily resolved in all circumstances, but sometimes subtle differences point to new subdivisions of a class. For example, the separation of $\mathrm{SNe}$ $\mathrm{Ib} / \mathrm{c}$ from SNe Ia is, by now, well-established and based on several independent signatures, including spectral differences near maximum, infrared light curves, and spectral evolutions at late phases (e.g., Harkness \& Wheeler 1990, and references therein). Nevertheless, because the differences near maximum light are subtle, the two subclasses were confused for many years. Now it appears that they have very different progenitor stars and unrelated explosion mechanisms. Indeed, the type $\mathrm{Ib} / \mathrm{c}$ subclass may have more in common with the type II supernovae than with SNe Ia.

More than any other subclass of supernovae, SNe Ia display a remarkable degree of similarity. Earlier investigations tried to subdivide the class based on differences in the light curve shapes (e.g., Pskovskii 1967, 1971, 1984; Barbon et al. 1973). These analyses, however, suffered from poor data and systematic errors and most of the old data sets are consistent with a uniform light evolution (e.g., Leibundgut et al. 1991a). Yet detailed observations over the last few years show that SNe Ia are not identical. Branch (1987) showed that SN 1984A had a significantly higher expansion velocity near maximum light than SN 1981B. Photometric variations were displayed by $\mathrm{SN}$ 1986G in NGC 5128 (Centaurus A), where the $B$ and $V$ light curves declined faster after maximum than those of SN 1981B (Phillips et al. 1987; Christiani et al. 1992) and the infrared light curves were also unusual (Frogel et al. 1987). Small but significant anomalies were observed in the optical spectrum of SN 1986G at maximum (Phillips et al. 1987) and later in SN 1990N (Leibundgut et al. 1991b). Much more spectacular deviations were revealed recently in the premaximum observations obtained of SN 1991T (Filippenko et al. 1992a; Ruiz-Lapuente et al. 1992; Phillips et al. 1992). These differences, however, disappeared within two weeks after maximum, and have been interpreted as possible abundance differences at the surface of the explosion (Jeffery et al. 1992). Interestingly, the $B$ 
and $V$ light curves of SN $1991 \mathrm{~T}$ resembled those of typical $\mathrm{SNe}$ Ia, and the peak luminosity also was normal (Phillips et al. 1992; see, however, Filippenko et al. 1992a; RuizLapuente et al. 1992). These variations among SNe Ia are I. poorly understood signatures of differences among progenitor stars and explosion mechanisms, but the unusual early time spectra of both SN 1990N and SN 1991T can be explained within the framework of an exploding $\mathrm{C}-\mathrm{O}$ white dwarf (Jeffery et al. 1992). As we will show below, SN 1991bg is an unusual case with significant spectroscopic and photometric peculiarities. Despite these differences, the main signatures of $\mathrm{SNe} \mathrm{Ia}$, i.e., lines of intermediate mass elements near maximum, iron lines at late phases, and the color and decline rates of the light curves at late epochs argue for a similar explosion mechanism for SN $1991 \mathrm{bg}$ and do not warrant creation of a new subdivision of the SN Ia class.

The discovery of SN 1991bg in the E1 galaxy NGC 4374 (Kosai et al. 1991) has added a truly peculiar event to the list of SNe Ia. SN 1991bg occurred 61" due south of the nucleus of NGC 4374, a member of the Virgo cluster of galaxies. It is the second supernova recorded in this galaxy after SN 1957B. Early reports recognized that SN 1991bg did not follow the evolution of most SNe Ia (Filippenko et al. 1991; Benetti et al. 1991; Della Valle et al. 1991; Phillips \& Hamuy 1991). The red color at maximum, the strength of the Si II lines, and the low velocities in the ejecta all pointed toward an event outside the normal range of SNe Ia (see also Filippenko et al. 1992b). Its discovery close to maximum makes it a good candidate for thorough testing of current ideas of SN Ia explosions in an extreme case. Our investigation centers on the question whether SN $1991 \mathrm{bg}$ can be understood as a variation of the SN Ia theme, or whether it demands a different physical explanation.

Although SN 1991bg is unusual, it may not be unique. Shortly after the peculiarities of SN 1991 bg had been recognized another supernova with a similar spectrum near maximum was discovered. A spectrum of SN 1992K in ESO 269-G57 shows strong correspondence with maximum light spectra of SN 1991bg (Hamuy et al. 1992).

Our observations of SN 1991bg are presented in Sec. 2. The light curves and spectra are discussed in Secs. 3 and 4. The discussion (Sec. 5) delineates the differences between this supernova and most other $\mathrm{SNe} I \mathrm{Ia}$ and examines some possible explanations for those differences. We conclude with Sec. 6.

\section{OBSERVATIONS}

The night following the discovery announcement of SN 1991bg (13 December 1991 (UT); Kosai et al. 1991) we initiated photometric and spectroscopic observations at the F. L. Whipple Observatory, the MMT, and CTIO. The location of the supernova in the sky (minimum hour angle 2 and 3 hours at Whipple Observatory and CTIO, respectively) restricted observations to morning twilight for the first few weeks, nevertheless we obtained several spectra and images. The photometric record of SN 1991bg is de-
TABLE 1. Photometry of SN $1991 \mathrm{bg}$.

\begin{tabular}{|c|c|c|c|c|c|c|c|c|}
\hline & Date & & $\begin{array}{c}\text { JD } \\
(2448000+) \\
\end{array}$ & $\overline{\mathrm{V}}$ & $\overline{B-V}$ & V-R & V-I & Notes \\
\hline \multirow[t]{14}{*}{1991} & $\overline{D e c}$ & 3.78 & 594.28 & 14.90 & - & - & - & 1 \\
\hline & & 9.84 & 600.36 & 14.50 & - & - & 一 & 2 \\
\hline & & 10.80 & 601.30 & 14.40 & - & - & - & 2 \\
\hline & & 10.80 & 601.30 & 14.30 & - & - & - & 2 \\
\hline & & 13.50 & 604.00 & 14.02 & 0.74 & 0.26 & 0.33 & 3 \\
\hline & & 14.50 & 605.00 & 13.96 & 0.85 & 0.33 & 0.48 & 3 \\
\hline & & 15.50 & 606.00 & 13.97 & 0.89 & 0.33 & 0.40 & 3 \\
\hline & & 16.50 & 607.00 & 14.01 & 0.95 & 0.36 & 0.55 & 3 \\
\hline & & 17.35 & 607.85 & 14.01 & 1.02 & - & - & 4 \\
\hline & & 18.34 & 608.84 & 14.06 & 1.16 & - & - & 4 \\
\hline & & 19.34 & 609.84 & 14.15 & 1.19 & - & - & 4 \\
\hline & & 20.33 & 610.83 & 14.24 & 1.35 & - & - & 4 \\
\hline & & 27.36 & 617.86 & 15.11 & 1.51 & - & - & 5 \\
\hline & & 30.36 & 620.86 & 15.36 & 1.42 & - & - & 6 \\
\hline \multirow[t]{22}{*}{1992} & Jan & 2.35 & 623.85 & 15.51 & 1.37 & - & - & 6 \\
\hline & & 4.34 & 625.84 & 15.62 & 1.32 & - & - & 7 \\
\hline & & 5.35 & 626.85 & 15.71 & 1.36 & - & - & 8 \\
\hline & & 6.34 & 627.84 & 15.74 & 1.31 & - & - & 8 \\
\hline & & 7.34 & 628.84 & 15.76 & 1.31 & - & - & 8 \\
\hline & & 10.35 & 631.85 & 15.88 & 1.29 & - & - & 9 \\
\hline & & 11.35 & 632.85 & 15.94 & 1.26 & - & - & 9 \\
\hline & & 20.34 & 641.84 & 16.36 & 1.02 & - & - & 10 \\
\hline & Feb & 4.32 & 656.82 & 16.78 & 0.95 & 0.27 & 0.72 & 11 \\
\hline & & 6.29 & 658.79 & 16.95 & 0.87 & 0.15 & 0.61 & 12 \\
\hline & & 8.33 & 660.83 & 17.01 & 0.86 & 0.11 & 0.70 & 12 \\
\hline & & 10.33 & 662.83 & 17.06 & 0.82 & 0.09 & 0.51 & 12 \\
\hline & & 21.29 & 673.79 & 17.53 & 0.72 & 0.12 & 0.86 & 13 \\
\hline & & 24.37 & 676.87 & 17.42 & 0.73 & - & - & 14 \\
\hline & & 26.30 & 678.80 & 17.58 & 0.68 & - & 1.02 & 15 \\
\hline & Mar & 6.41 & 687.91 & 17.96 & - & - & - & 16 \\
\hline & & 7.41 & 688.91 & 17.92 & 0.69 & - & - & 17 \\
\hline & & 11.24 & 692.74 & 18.00 & 0.53 & - & - & 18 \\
\hline & Apr & 8.19 & 720.69 & 18.49 & 0.39 & - & - & 19 \\
\hline & & 30.29 & 742.79 & 19.48 & 0.20 & - & - & 20 \\
\hline & May & 19.98 & 762.48 & 19.74 & - & 1.35 & 1.70 & 21 \\
\hline & Jun & 3.29 & 776.79 & 20.03 & -0.07 & - & - & 20 \\
\hline 1991 & Dec & 27.34 & 617.84 & 15.05 & 1.39 & - & - & 22 \\
\hline \multirow[t]{2}{*}{1992} & Jan & 7.34 & 628.84 & 15.82 & 1.22 & - & - & 23 \\
\hline & Feb & 5.31 & 657.81 & 16.98 & 0.87 & - & - & 23 \\
\hline
\end{tabular}

1 photovisual; Y. Kushida (1

2 photoelectric; T. Kato (Kosai et al. 1991)

$3 \mathrm{Mt}$. Hopkins $1.2 \mathrm{~m}$ SAO telescope with Loral CCD; B. Leibundgut

4 CTIO $0.9 \mathrm{~m}$ with Tek 1024 (\#2); N. Caldwell

5 CTIO $0.9 \mathrm{~m}$ with Tek 1024 (\#2); L. Gonzalez

6 CTIO $0.9 \mathrm{~m}$ with Tek 1024 (\#2); D. Rehner

7 CTIO $0.9 \mathrm{~m}$ with Tek 1024 (\#2); A. Walker

8 CTIO 0.9m with Tek 1024 (\#2); R. Schommer

9 CTIO $0.9 \mathrm{~m}$ with Tek 1024 (\#2); J. Parker

10 CTIO $0.9 \mathrm{~m}$ with Tek 512 (\#4); T. Barnes

11 CTIO $0.9 \mathrm{~m}$ with TI (\#3); P.Schmidtke

12 CTIO $0.9 \mathrm{~m}$ with Tek 2048 (\#1); P. Hintzen and K. Cheng

13 CTIO 4m with Tek 1024 (\#2); N. Suntzeff

14 CTIO $0.9 \mathrm{~m}$ with Tek 1024 (\#1); L. Siciliano

15 CTIO $0.9 \mathrm{~m}$ with Tek 1024 (\#1); N. Suntzeff

16 CTIO 4m with Tek 1024 (\#2); N. Suntzeff and M. Navarrete 17 KPNO $2.1 \mathrm{~m}$ with T1KA; B. Leibundgut

18 CTIO 4m with Tek 1024 (\#2); A. Walker and R. Schommer 19 CTIO $0.9 \mathrm{~m}$ with Tek 1024 (\#2); Y. Kim 20 KPNO $2.1 \mathrm{~m}$ with T1KA; A. Porter

21 CTIO $0.9 \mathrm{~m}$ with Tek 1024 (\#1); G. Williger

22 CTIO $1.5 \mathrm{~m}$ CSCCD/GEC10 (spectrophotometry); M. Phillips 23 CTIO 4m CSCCD/Reticon (spectrophotometry); M. Hamuy

tailed in Table 1 . We concentrated on the $B$ and $V$ filters and only occasional $R$ and $I$ observations were obtained. The zero point of the photometry is based on several photometric nights at Mt. Hopkins and at CTIO, during which standard stars from the Selected Areas (Landolt 1983) and the E-Regions (Graham 1982) were observed. These data 
TABLE 2. Photometry of comparison stars in the field of SN 1991bg.

\begin{tabular}{|c|c|c|c|c|c|c|c|c|c|c|c|c|}
\hline designation & V & $\sigma$ & II & $\mathrm{B}-\mathrm{V}$ & $\sigma$ & $\mathrm{n}$ & $\mathrm{V}-\mathrm{R}$ & $\sigma$ & 11 & V-1 & $\sigma$ & $\mathrm{n}$ \\
\hline $\mathrm{A}$ & 14.59 & 0.04 & 12 & 1.42 & 0.03 & 12 & 0.87 & 0.02 & 2 & 1.73 & 0.04 & 2 \\
\hline B & 16.62 & 0.03 & 13 & 0.50 & 0.06 & 13 & 0.35 & 0.06 & 3 & 0.56 & 0.04 & 3 \\
\hline C & 15.95 & 0.04 & 8 & 0.60 & 0.04 & 8 & -- & & & $-\cdot$ & $\ldots$ & -- \\
\hline D & 15.42 & 0.04 & 8 & 1.02 & 0.02 & 8 & $\ldots$ & $\cdots$ & ... & - & -- & - \\
\hline $\mathrm{E}$ & 16.17 & 0.04 & 11 & 0.82 & 0.03 & 11 & 0.42 & 0.08 & 3 & 0.89 & 0.02 & 3 \\
\hline $\mathrm{F}$ & 17.25 & 0.07 & 8 & 1.09 & 0.07 & 8 & $\ldots$ & -. & - & - & $\ldots$ & -- \\
\hline G & 17.19 & 0.03 & 3 & 1.40 & 0.10 & 3 & - & - & ... & ... & - & $-\cdot$ \\
\hline H & 15.82 & $\ldots$ & 1 & 1.16 & - & 1 & $\ldots$ & $-\ldots$ & - & -- & $\cdots$ & .... \\
\hline I & 16.76 & 0.06 & 4 & 0.62 & 0.07 & 4 & 0.31 & 0.0 .1 & 3 & $0.6 \pi$ & 0.00 & 3 \\
\hline $\mathrm{J}$ & 12.31 & 0.29 & 6 & 0.38 & 0.23 & 6 & $\ldots$ & & & - & - & - \\
\hline $\mathrm{K}$ & 16.60 & 0.06 & 4 & 0.52 & 0.07 & 4 & 0.38 & 0.06 & 3 & 0.75 & 0.05 & 3 \\
\hline L & 17.01 & 0.06 & 3 & $0.4 \pi$ & 0.07 & 3 & 0.30 & 0.13 & 3 & 0.71 & 0.12 & 3 \\
\hline $\mathrm{M}$ & 16.69 & 0.02 & 3 & 0.53 & 0.03 & 3 & 0.31 & 0.02 & 3 & 0.61 & 0.10 & 3 \\
\hline
\end{tabular}

were used to calibrate local standards in the field of SN 1991 bg which are identified in Fig. 1 (Plate 8). The Mt. Hopkins and CTIO data sets were reduced completely independently using aperture photometry within IRAF. ${ }^{6}$ Comparing the magnitudes and colors of stars in both data sets of images revealed no systematic difference $(\leqslant 0 \mathrm{~m} 02)$ between them. Table 2 gives the magnitudes and colors of the local standard stars based on the combined data sets. The errors given are the standard deviations of the measurements of each star, which typically amount to less than $0.05 \mathrm{mag}$ for the brighter stars and up to $0.1 \mathrm{mag}$ for stars as faint as 17 th mag. The final light curves (Fig. 2) are based on differential photometry of the supernova carried out with respect to the local standards. The supernova is located at about 1.4 effective radii of the elliptical galaxy (Burstein et al. 1987), and galaxy background clearly affects the photometry (Fig. 1). Hence, special attention was given to determining the background. Subtracting the background by fitting the surface brightness of the galaxy or subtracting a smoothed image where the stellar objects had been removed did not yield significant improvements over aperture photometry. Around maximum light all the background corrections correspond to less than $0.05 \mathrm{mag}$ in all filters. At later epochs the background contamination is a larger fraction of the supernova light, but the background subtraction in an annulus around the supernova corrects for the smooth galaxy brightness to within 0.1 mag. We are confident that the effects of background contamination on the shapes of the light curves (Boisseau \& Wheeler 1991) are negligible during the period covered by our observations.

Our spectroscopic observations of SN 1991bg start near maximum, which occurred on 14.7 December in $V$ (see below), and continue well into the nebular phase of the supernova. Low-dispersion spectra were obtained at CTIO with the 4 and $1.5 \mathrm{~m}$ telescopes, at the MMT, and with the Tillinghast $1.5 \mathrm{~m}$ telescope on Mt. Hopkins. A log of the spectroscopic observations can be found in Table 3. All spectra were wavelength calibrated and fluxed with IRAF tasks to remove the instrumental sensitivity variations. Spectra from CTIO were further observed with two slit widths ( 2 " and $\left.10^{\prime \prime}\right)$ to assure spectrophotometry on photometric nights. Subtraction of background light was accomplished using pixels adjacent to the supernova in the

${ }^{6}$ IRAF is distributed by National Optical Astronomy Observatories.
TABLE 3. Spectroscopy of SN $1991 \mathrm{bg}$.

\begin{tabular}{|c|c|c|c|c|c|c|}
\hline \multicolumn{3}{|c|}{ Date } & \multirow{2}{*}{$\begin{array}{c}\text { JD } \\
(2448000+) \\
603.99\end{array}$} & \multirow{2}{*}{$\begin{array}{c}\begin{array}{c}\text { Wavelength } \\
\text { coverage }\end{array} \\
4650-7050\end{array}$} & \multirow{2}{*}{$\begin{array}{c}\begin{array}{c}\text { Telescope } \\
\text { Instrument }\end{array} \\
1\end{array}$} & \multirow{2}{*}{$\begin{array}{c}\text { Observer } \\
a\end{array}$} \\
\hline 1991 & Dec & 13.49 & & & & \\
\hline & & 15.36 & 605.86 & $3200-7500$ & 2 & b \\
\hline & & 15.52 & 606.02 & $6200-6800$ & 3 & c \\
\hline & & 16.51 & 607.01 & $6200-6800$ & 3 & c \\
\hline & & 27.34 & 617.84 & $3000-7750$ & 4 & d \\
\hline & & 28.34 & 618.84 & $5150-9650$ & 4 & e \\
\hline & & 31.50 & 622.00 & $4600-7200$ & 1 & $f$ \\
\hline & & 31.56 & 622.06 & $3200-7900$ & 5 & $\mathrm{~g}$ \\
\hline \multirow[t]{7}{*}{1992} & Jan & 7.34 & 628.84 & $3250-7500$ & 2 & $\mathrm{~h}$ \\
\hline & & 8.34 & 629.84 & $6300-10650$ & 2 & $\mathrm{~h}$ \\
\hline & & 14.40 & 635.90 & $3700-8780$ & 3 & $\mathrm{i}$ \\
\hline & & 28.30 & 649.80 & $3150-7800$ & 4 & $\mathrm{j}$ \\
\hline & Feb & 5.31 & 657.81 & $3200-7500$ & 2 & $\mathrm{~h}$ \\
\hline & Mar & 7.28 & 688.78 & $3700-8000$ & 3 & k \\
\hline & Apr & 4.17 & 716.67 & $3250-7500$ & 2 & $\mathrm{~h}$ \\
\hline \multicolumn{7}{|c|}{ Notes to TABLE 3} \\
\hline \multicolumn{4}{|c|}{$\begin{array}{l}1 \text { 1.5m Tillinghast; z-machine } \\
2 \text { CTIO 4m; CSCCD/Reticon } \\
3 \text { MMT; RedChannel/TI } 5 \\
4 \text { CTIO } 1.5 \mathrm{~m} \text {; CSCCD/GEC } 10 \\
5 \text { MMT; BlueChannel/Reticon }\end{array}$} & \multicolumn{3}{|c|}{$\begin{array}{l}\text { a J. Peters and B. Leibundgut } \\
\text { b J. Maza } \\
\text { c B. Whitney and M. Gomez } \\
\text { d P. Ugarte } \\
\text { e M. Phillips } \\
\text { f J. Peters } \\
\text { g R. Marzke } \\
\text { h M. Hamuy } \\
\text { i B. Schmidt } \\
\text { j M. Hamuy and R. Williams } \\
\text { k F. Chaffee and C. Foltz }\end{array}$} \\
\hline
\end{tabular}

long slit images. (The spectral evolution of SN $1991 \mathrm{bg}$ is displayed in Fig. 4.)

\section{LIGHT CURVES AND COLOR CURVES}

Figure 2 displays our CCD photometry together with synthetic magnitudes derived from the CTIO spectrophotometry. Also plotted are the premaximum photovisual magnitude estimates of Kushida and the $V$ magnitude measured photoelectrically by Kato (Kosai et al. 1991). Our earliest photometry was evidently obtained close to maximum light. Maximum brightness in $V$ occurred on 1991 December $14.7 \pm 0.5$ days (JD 2448605.3 \pm 0.5 ) at $m_{V}=13.95 \pm 0.02$ in excellent agreement with the values found by Filippenko et al. (1992b). The maximum in $B$ is not covered by our data, and we can only estimate that the peak was reached at least one day earlier than in $V$. Other SNe Ia show a typical time interval of 1 to 2 days between $B$ and $V$ maxima (Phillips et al. 1987; Leibundgut 1988; Hamuy et al. 1991; Leibundgut et al. 1991b; Phillips et al. 1992 ), but due to the unusually red color of SN 1991bg, establishing the $B$ maximum date based on comparison with other $\mathrm{SNe}$ Ia does not seem prudent. Instead we will deviate from the usual practice of using the $B$ maximum as the time zero point and describe phases for this supernova relative to the $V$ maximum. Figure 2 also shows the light curve templates for SNe Ia of Leibundgut (1988) which are a good representation of most $\mathrm{SNe}$ Ia (Leibundgut et al. 1991a,b; Hamuy et al. 1991; Phillips et al. 1992; Wells et al. 1992). The $V$ template is matched with the observations at the maximum. Since the $B$ maximum is not determined we matched the $B$ template with the first observation available. It is clear from the figure that the templates are not an adequate representation of the light curves of SN $1991 \mathrm{bg}$. The templates represent the photom- 


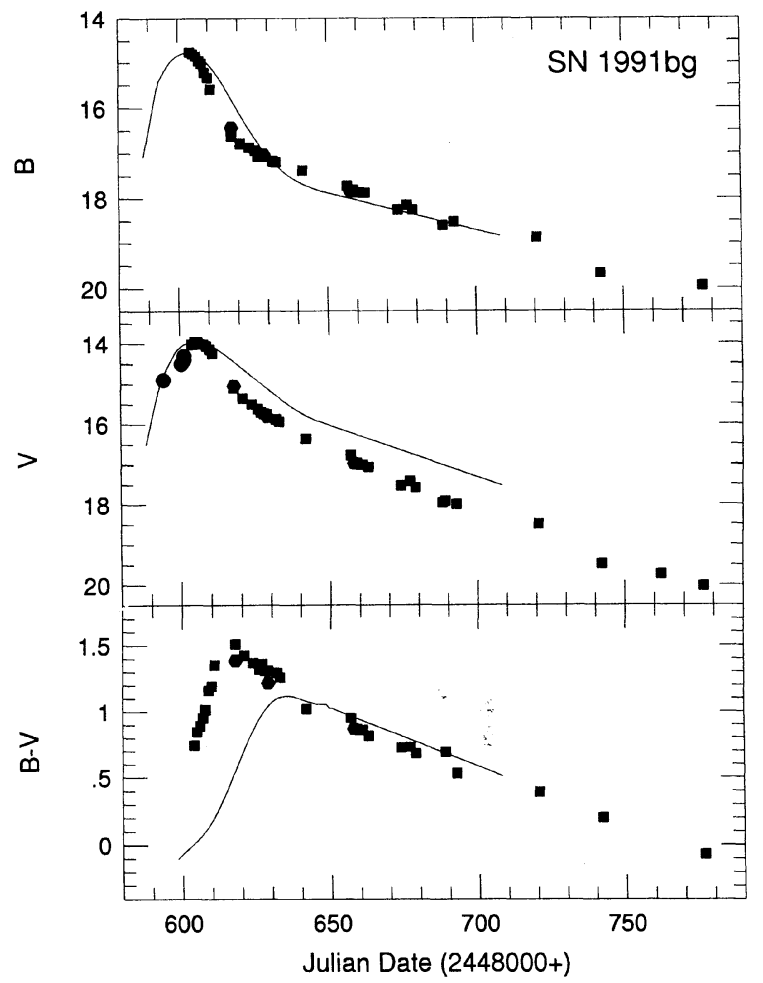

FIG. 2. The $B$ (top) and $V$ (bottom) light curves of SN $1991 \mathrm{bg}$. The squares are CCD photometry from CTIO and Mt. Hopkins and the hexagons spectrophotometry from CTIO. Also shown as filled circles are the premaximum observations reported by Kosai et al. (1991). The template light curves for SNe Ia are shown for comparison.

etry of most well-observed SNe Ia to better than $0 \mathrm{~m} 2$ (Leibundgut 1991), with SN 1986 G and SN 1939B being the most notable exceptions. The light curves of SN 1991bg fall by 2.05 and $1.42 \mathrm{mag}$ during the first 15 days past maximum in $B$ and $V$, respectively, while the declines in the templates amount to only 1.22 and 0.64 in the same filters. The $B$ and $V$ light curves of SN $1991 \mathrm{bg}$ decline between 15 and 40 days at rates of $0.029 \pm 0.003 \mathrm{mag} / \mathrm{day}$ and $0.047 \pm 0.001$ for $B$ and $V$, respectively. The light curves change slope again between $35(B)$ and $45(V)$ days after $V$ maximum comparable to the templates with change slope $44(B)$ and 41 days $(V)$ after $V$ maximum. The decline rates after 50 days past $V$ maximum are 0.021 \pm 0.001 in $B$ and $0.028 \pm 0.001$ in $V$ for SN 1991bg which are marginally larger than the rates of the templates $(0.017$ in $B$ and 0.026 in $V$; Leibundgut 1988).

The premaximum observations of Kushida and Kato suggest a faster rise time for SN 1991bg than for the template. If this is correct, then SN 1991bg had a much narrower peak than other type Ia supernovae. The width of the $V$ light curve measured one magnitude below maximum corresponds to $\sim 23$ days for $\mathrm{SN} 1991 \mathrm{bg}$ compared to $\sim 33$ days for the $V$ template.

The $B-V$ color evolution is displayed in the bottom panel of Fig. 2. In this diagram, the peculiarities described above are most obvious. We have plotted the comparison

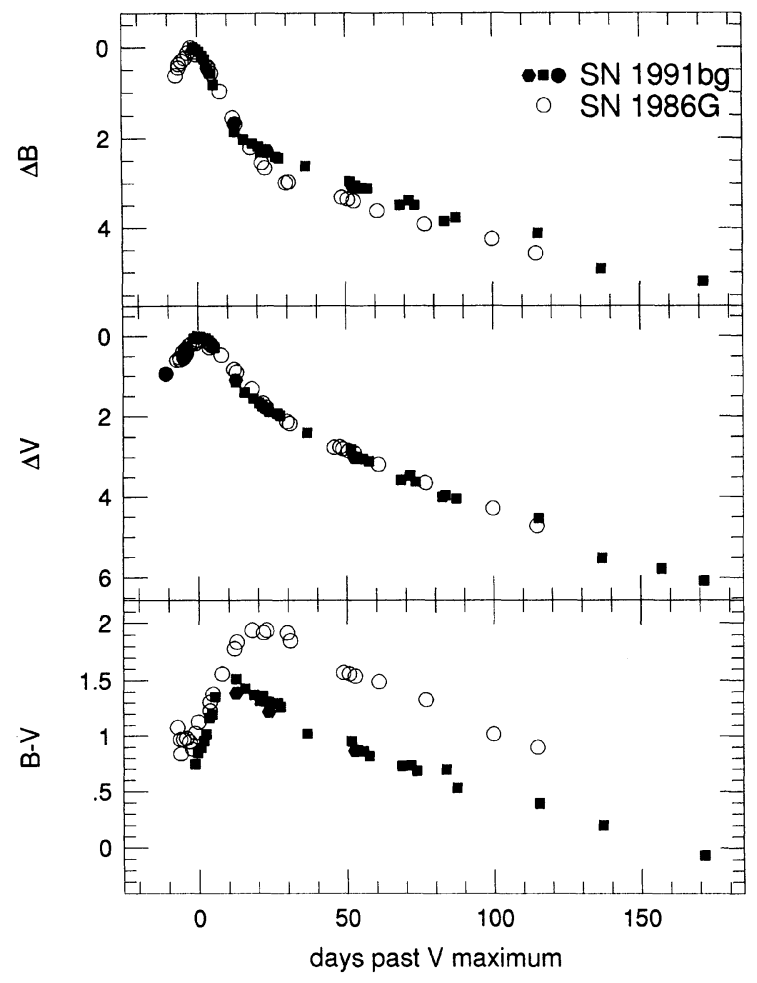

FIG. 3. Comparison of the light curves of SN 1991bg and SN 1986G. The two light curves are matched at maximum. Note the steeper declines in SN 1991bg and the shorter decline phase after maximum.

template with an assumed color at maximum of $B-V$ $=0.0$ (Hamuy et al. 1991), fixing the phases by the occurrence of the $V$ maximum. The unusually red color of $\mathrm{SN}$ $1991 \mathrm{bg}$ at early epochs is strikingly evident in this figure. Note also that the maximum in the color curve is reached after only 15 days, while the template rises for about 30 days. The $B-V$ color evolution at late phases resembles most other SNe Ia. SN 1991bg is as blue as the template at these epochs. The slope of the curve is $-0.010 \pm 0.001$ $\mathrm{mag} /$ day which is almost identical with the slope of the template of -0.009 .

The fast decline of SN $1991 \mathrm{bg}$ after maximum resembles the well-observed SN 1986G (Phillips et al. 1987; Frogel et al. 1987). Comparison of the light and color curves of the two objects, however, reveals distinct differences (Fig. 3 ). While the light curves of SN 1986G bend at $\sim 30$ days past maximum, SN $1991 \mathrm{bg}$ changes slope 15 days earlier. At an epoch of 15 days, SN $1991 \mathrm{bg}$ had declined by $\sim 0.2$ mag more in $B$ than SN $1986 \mathrm{G}$, and by $\sim 0.6$ mag more in $V$. Thus, SN $1991 \mathrm{bg}$ had an even steeper decline after maximum than SN 1986G. The rates of decline in the exponential tails of SN $1986 \mathrm{G}$ were $0.019 \pm 0.001$ in $B$ and $0.029 \pm 0.0003$ in $V$. The latter is almost identical with SN $1991 \mathrm{bg}$, while the decline in $B$ is intermediate between the templates and SN 1991bg. The lower panel of Fig. 3 is particularly revealing of the differences between the two supernovae. The phase of redward evolution of SN 1991bg 
is much shorter than that of SN $1986 \mathrm{G}$ and the slope of the redward evolution is not the same. SN 1991 bg changes the $B-V$ color from 0.75 to 1.5 in the 12 days past $V$ maximum when the peak in the $B-V$ curve is reached, whereas SN $1986 \mathrm{G}$ evolves from 1.0 to 1.9 in 21 days. The decline of SN 1991bg at late times, however, is again comparable to the templates and SN 1986G $(-0.011 \pm 0.0004)$.

Another supernova with a fast decline after maximum is SN 1939B in NGC 4621 (Shapley 1939; Hoffleit 1939; Campbell 1939; Baade 1964) which dropped by 3.3 mag in the photographic band during the first 15 days. This decline is even larger than those observed in the $B$ band for SN 1986G or SN 1991bg.

We searched the atlas of SNe Ia light curves (Leibundgut et al. 1991a) for other events with such a steep initial decline. Such a search is hampered by scanty photometry for many supernovae and observational problems in many of the data sets. Only two supernovae were found with possible longer or steeper decline phases than the templates. SN 1963P appears to have faded a little faster in $B$ than the template suggests (Bertola et al. 1965), and SN 1984A (Kimeridze \& Tsvetkov 1986; Barbon et al. 1989) might have declined longer than the template, although the data are quite noisy.

\section{SPECTRAL EVOLUTION}

Beginning with the first observations SN 1991bg displayed several peculiarities in its spectrum. The strong Si II $\left(\lambda_{0}=6355 \AA\right)$ absorption at $6150 \AA$ clearly marks this event as a type Ia supernova (Kirshner et al. 1991; Filippenko et al. 1991, 1992b), but the atypical strengths of other lines were noted immediately (Filippenko et al. 1991; Phillips \& Hamuy 1991). In the following we adopt the line identifications for SNe Ia of Jeffery et al. (1992) which agree with those given by Filippenko et al. (1992a,b); Ruiz-Lapuente et al. (1992) and, with the exception of Fe III lines, the earlier ones of Branch et al. (1983, 1985) and Harkness (1991a,b). At late phases the identifications are drawn from Axelrod (1980a). The line identifications for SN 1991bg cannot be definitive, since they are not the result of spectral synthesis calculations. We chose to label the minima with lowercase and maxima in uppercase characters in order to include unidentified lines in the discussion. This is potentially dangerous as it is not clear which features are in absorption and which in emission, and we expect a shift from absorption to emission lines as the supernova evolves.

The spectral evolution of SN 1991bg is illustrated in Fig. 4. The overall appearance of the supernova changed between the two early spectra near peak light and the third one which was obtained at an epoch $\sim 2$ weeks past $V$ maximum. By this time the broad emission features which are ordinarily observed in SNe Ia at late phases (age $\geqslant 40$ days) have already begun to appear.

The spectra at maximum light are dominated by lines of $\mathrm{Ca}$ II, Si II, and S II as identified in Table 4. Ca II H \& $\mathrm{K}$ are seen strong in emission while the absorption component of this scattering line is present but poorly defined due

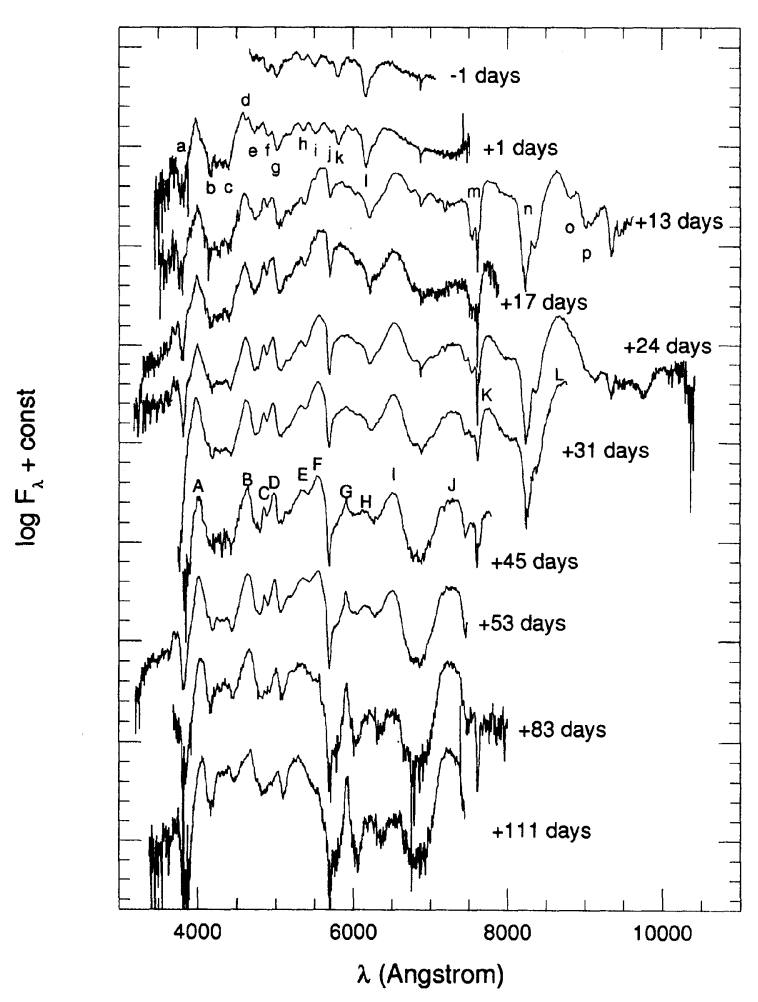

FIG. 4. Spectral evolution of SN 1991bg. The spectra have been displaced on the ordinate by arbitrary amounts for clarity. Absorption lines are labeled by lowercase letters, emission lines with uppercase letters.

to the low signal-to-noise. We tentatively identify feature c with the usual $\mathrm{Mg}$ II and Fe III absorptions observed in other SNe Ia (e.g., Jeffery et al. 1992), although the whole wavelength region between $4100-4400 \AA$ appears unusual for a type Ia event. Filippenko et al. (1992b) fit this part of the spectrum with $\mathrm{Ti}$ II (for our feature b) and $\mathrm{Mg}$ II (feature $c$ ). This region remains depressed well after a month past maximum and is filled in by emission only after $\sim 50$ days. The maximum light spectra are dominated by many lines of intermediate mass elements and the Fe III lines ( $\lambda_{0}=4404 \AA$ and $\lambda_{0}=5129$ multiplets) identified in the spectra of SN 1991T (Filippenko et al. 1992a; RuizLapuente et al. 1992; Jeffery et al. 1992). Both Fe III absorptions appear quite strong compared to other SNe Ia (see, however, Filippenko et al. $1992 \mathrm{~b}$ for a different interpretation). All S II and Si II lines are relatively well-defined and sharp. The Si II absorption at $5800 \AA$ (designated with k) is stronger than in many other SNe Ia at comparable epochs. The characteristic Si II absorption at $6150 \AA$ appears to be blended with another, unidentified, line at the red end. A possible identification is C II $(6580 \AA)$ with an expansion velocity of about $14500 \mathrm{~km} \mathrm{~s}^{-1}$. Alternatively, this feature could be $\mathrm{H} \alpha$ at a velocity of $\sim 14000 \mathrm{~km} \mathrm{~s}^{-1}$. Such an identification, however, appears rather unlikely for a type Ia supernova. The expansion velocity inferred for the Si II absorption at $6150 \AA$ is $\sim 10600 \mathrm{~km} \mathrm{~s}^{-1}$, when corrected for the galaxy redshift, which is comparable with 
TABLE 4. Line absorptions in spectra of SN 1991bg.

\begin{tabular}{|c|c|c|c|c|c|c|c|c|c|c|c|c|c|c|}
\hline \multirow{4}{*}{$\begin{array}{c}\text { Line } \\
\text { designation }\end{array}$} & \multirow{4}{*}{$\begin{array}{l}\text { Line } \\
\text { identification }\end{array}$} & \multicolumn{13}{|c|}{ Date } \\
\hline & & \multirow{2}{*}{\multicolumn{6}{|c|}{$\begin{array}{c}1991 \\
\text { December }\end{array}$}} & \multicolumn{7}{|c|}{1992} \\
\hline & & & & & & & & \multicolumn{4}{|c|}{ January } & \multirow{2}{*}{$\begin{array}{c}\text { February } \\
5.31 \\
(+53)\end{array}$} & \multirow{2}{*}{$\begin{array}{c}\text { March } \\
7.28 \\
(+83) \\
\end{array}$} & \multirow{2}{*}{$\begin{array}{c}\text { April } \\
4.17 \\
(+111) \\
\end{array}$} \\
\hline & & $\begin{array}{l}13.49 \\
(-1)^{a}\end{array}$ & $\begin{array}{l}15.36 \\
(+1)\end{array}$ & $\begin{array}{l}27.34 \\
(+13)\end{array}$ & $\begin{array}{l}28.34 \\
(+14)\end{array}$ & $\begin{array}{l}31.50 \\
(+17)\end{array}$ & $\begin{array}{l}31.56 \\
(+17)\end{array}$ & $\begin{array}{c}7.34 \\
(+24) \\
\end{array}$ & $\begin{array}{c}8.34 \\
(+25) \\
\end{array}$ & $\begin{array}{l}14.40 \\
(+31) \\
\end{array}$ & $\begin{array}{l}28.30 \\
(+45) \\
\end{array}$ & & & \\
\hline $\mathrm{a}$ & $\mathrm{Ca}$ II $(\mathrm{H}+\mathrm{K})$ & - & 3800 & 3780: & - & - & 3800 & 3820 & - & - & - & 3810 & 3840 & - \\
\hline $\mathrm{b}$ & - & - & 4160 & 4150: & - & - & 4170: & 4180 & - & 4170: & 4170: & 4200 & 4160 & 4170 \\
\hline c & Mg II; Fe III & - & 4390 & 4400: & - & - & 4410: & 4420: & - & 4420: & 4430: & 4440 & 4440 & 4450: \\
\hline $\mathrm{d}$ & Si III & - & 4610 & - & - & - & - & - & - & - & - & - & - & - \\
\hline e & - (blend) & 4720 & 4720 & 4740 & - & 4750: & 4720 & 4750 & - & 4760 & 4780: & 4780: & - & - \\
\hline$f$ & Si II & 4900 & 4900 & 4890 & - & 4890 & 4890 & 4890 & - & 4900 & 4890 & 4900 & - & - \\
\hline \multirow[t]{2}{*}{ g } & Fe III (blend) & 5010 & 5030 & $5060:$ & - & 5040 & 5070 & 5070 & - & 5060 & 5060 & 5080 & 5100 & 5100 \\
\hline & & - & 5150 & $5210:$ & - & 5140: & 5200 & 5200 & - & 5180 & 5180 & 5220 & - & - \\
\hline $\mathrm{h}$ & S II (blend) & 5350 & 5340 & $5380:$ & 5380 & 5380 & 5380 & 5390 & - & 5400 & 5420 & 5430 & - & - \\
\hline $\mathrm{i}$ & S II & 5500 & 5510 & - & - & - & - & - & - & - & - & - & - & - \\
\hline \multirow[t]{2}{*}{$\mathrm{j}$} & Si II (blend) & 5700 & 5710 & 5700 & 5710 & 5700 & 5700 & 5700 & - & 5700 & 5700 & 5700 & 5700: & - \\
\hline & & - & - & - & - & 5760: & 5780 & 5790 & - & 5790 & 5780 & 5790 & 5810 & - \\
\hline $\mathrm{k}$ & Si II & 5800 & 5810 & - & - & - & - & - & - & - & - & - & - & - \\
\hline \multirow[t]{2}{*}{1} & Si II (blend) & 6160 & 6170 & 6200 & 6200 & 6210 & 6210 & 6210 & - & 6230 & 6280: & $6280:$ & - & - \\
\hline & & 6240 & 6270 & 6270 & 6250 & 6290: & 6310 & 6320 & - & - & - & - & - & - \\
\hline \multirow[t]{2}{*}{$\mathrm{m}$} & O I & - & - & - & - & - & - & - & 7450 & 7440 & 7440 & - & - & - \\
\hline & & - & - & 7530 & 7540 & - & $7550:$ & - & 7530 & 7540 & $7540:$ & - & - & - \\
\hline \multirow[t]{2}{*}{$\mathrm{n}$} & $\mathrm{Ca}$ II & - & - & - & 8220 & - & - & - & 8230 & 8220 & - & - & - & - \\
\hline & & - & - & - & 8350 & - & - & - & 8370 & 8400 & - & - & - & - \\
\hline o & C I? & - & - & - & 8810 & - & - & - & - & - & - & - & - & - \\
\hline $\mathrm{p}$ & - & - & - & - & 9000 & - & - & - & - & - & - & - & - & - \\
\hline
\end{tabular}

${ }^{a}$ Phase of observation in days past assumed V maximum (JD 2448605.3)

velocities of other well-observed $\mathrm{SNe}$ Ia at maximum (Branch et al. 1988; Leibundgut et al. 1991b; Phillips et al. 1992). The velocity of the Ca II H \& K absorption minimum corresponds to $\sim 12000 \mathrm{~km} \mathrm{~s}^{-1}$, which is much slower than observed for SN $1990 \mathrm{~N}\left(\sim 21000 \mathrm{~km} \mathrm{~s}^{-1}\right.$; Leibundgut et al. 1991b) but comparable to SN 1991T (12000-13000 $\mathrm{km} \mathrm{s}^{-1}$; Phillips et al. 1992).

An ultraviolet spectrum was obtained with $I U E$ almost exactly at $V$ maximum on 14.8 December (UT). The signal attained in an exposure of 150 minutes integration was very low. We measure an integrated flux between 2400 and $3150 \AA$ of about $6.5 \times 10^{-13} \mathrm{erg} \mathrm{s}^{-1} \mathrm{~cm}^{-2}$. No features are apparent in the spectrum. The broad emission peak near $2950 \AA$ observed in other SNe Ia (Blair \& Panagia 1987) is not discernible due to the low signal in the spectrum. There is, however, an increase of flux with increasing wavelength consistent with the observations of other SNe Ia. The UV flux between 2400 and $3150 \AA$ is about 14 times weaker in SN 1991bg than for SN $1990 \mathrm{~N}$ at a comparable epoch (Leibundgut et al. 1991b), while the observed flux ratios between SN 1991bg and SN $1990 \mathrm{~N}$ are $\sim 1 / 7$ and $\sim 1 / 4$ in $B$ and $V$, respectively.

A moderate resolution $[\Delta \lambda(\mathrm{FWHM}) \approx 3 \AA]$ spectrum of SN $1991 \mathrm{bg}$ covering the wavelength range from 6200 to $6800 \AA$ was obtained near maximum. No narrow line features are obvious, although shallow broad absorptions at 6420,6650 , and $6740 \AA$ appear to be present. The spectrum shows no signs of a possible scattering line due to $\mathrm{H} \alpha$ in accord with the observations in other SNe Ia. We also searched for a narrow absorption line due to possible hydrogen absorption in NGC 4374, as reported in the case of SN 1990M (NGC 5493; Polcaro \& Viotti 1991), but could not detect any trace of such an absorption.

Two weeks after the $V$ maximum the S II and Si II lines either disappeared or drastically changed their appearance. The same is true for the Fe III absorptions. The Ca II lines and the $6150 \AA$ absorption of Si II remain strong. P Cygni absorptions of $\mathrm{O} \mathrm{I}$ and $\mathrm{Ca}$ II are observed in the near infrared, a region not covered by the earlier spectra. The absorptions $o$ and $p$ are of unknown origin; a possible identification for the absorption o is C II (9111 $\AA$ ). This is the equivalent transition to the O I (7774 $\AA$ ) line observed as absorption $\mathrm{m}$. Both troughs $\mathrm{o}$ and $\mathrm{p}$ disappeared 10 days later with only the smooth wing of the $\mathrm{Ca}$ II emission blend still visible. The evolution of the supernova is much slower after day 13. The appearance of the spectrum at 30 days is not very different from the one at 13 days. Following Kirshner \& Oke (1975) and Axelrod (1980a,b) we identify most of the apparent emission features with [Fe II] and [Fe III] multiplets, although the Ca II ( $\mathrm{H} \& \mathrm{~K}$ ) scattering line persists over the entire period of our observations. From Table 4 a break in the evolution of the supernova around day 13 is also evident; while many absorptions shift to redder wavelength before this phase, most of the lines show little displacement after 13 days. This is also observed in normal SNe Ia, but the slow down in the evolution occurred much earlier in SN 1991bg. As discussed below, this early change in the spectrum and the rapid evolution in the light curve are important clues to the physical difference between SN 1991bg and other SNe Ia. The identifications given in Table 4 refer to the maximum phase and are not applicable at later epochs. The Si III line at $4600 \AA$ (d) and the Si II feature at $5800 \AA$ (k) are not discernible after maximum light. A slight secular trend of the emission to longer wavelengths can be deduced from Table 5 , but most measurements are uncertain due to the breadth of the features. The emission $F$ increases in strength until day $\sim 30$ after which it decays and blends with emission $\mathrm{E}$ 
TABLE 5. Line emissions in spectra of SN 1991bg.

\begin{tabular}{|c|c|c|c|c|c|c|c|c|c|c|c|c|c|c|}
\hline \multirow{4}{*}{$\begin{array}{c}\text { Line } \\
\text { designation }\end{array}$} & \multirow{4}{*}{$\begin{array}{c}\text { Line } \\
\text { identification }\end{array}$} & \multicolumn{13}{|c|}{ Date } \\
\hline & & \multirow{2}{*}{\multicolumn{6}{|c|}{$\begin{array}{c}1991 \\
\text { December }\end{array}$}} & \multicolumn{7}{|c|}{1992} \\
\hline & & & & & & & & & Jan & Iary & & February & March & April \\
\hline & & $\begin{array}{l}13.49 \\
(-1)^{a}\end{array}$ & $\begin{array}{l}15.36 \\
(+1)\end{array}$ & $\begin{array}{l}27.34 \\
(+13)\end{array}$ & $\begin{array}{l}28.34 \\
(+14)\end{array}$ & $\begin{array}{l}31.50 \\
(+17)\end{array}$ & $\begin{array}{l}31.56 \\
(+17)\end{array}$ & $\begin{array}{c}7.34 \\
(+24) \\
\end{array}$ & $\begin{array}{c}8.34 \\
(+25) \\
\end{array}$ & $\begin{array}{l}14.40 \\
(+31) \\
\end{array}$ & $\begin{array}{r}28.30 \\
(+45) \\
\end{array}$ & $\begin{array}{c}5.31 \\
(+53)\end{array}$ & $\begin{array}{c}7.28 \\
(+83)\end{array}$ & $\begin{array}{c}4.17 \\
(+111) \\
\end{array}$ \\
\hline \multirow[t]{2}{*}{$\mathrm{A}$} & $\mathrm{Ca}$ II $(\mathrm{H}+\mathrm{K})$ & - & 3970 & 4000 & - & - & 3990 & 4000 & - & 3980 & 4010: & 4020 & 4020 & 4060: \\
\hline & & - & 4060 & - & - & - & - & - & - & - & 4080: & 4160: & - & - \\
\hline $\mathrm{B}$ & Fe III & - & 4590 & 4600 & - & - & 4610 & 4620 & - & 4620 & 4630: & 4640 & 4630 & 4660 \\
\hline $\mathrm{C}$ & - & - & 4830 & 4850 & - & 4840: & 4850 & 4850 & - & 4850 & 4860 & 4860 & - & - \\
\hline D & Fe III? & - & 4940 & 4950 & - & 4960 & 4950 & 4970 & - & 4980 & 4990 & 5000 & 4980: & $5020:$ \\
\hline $\mathrm{E}$ & - & 5270 & 5290 & 5310 & - & 5320 & 5320 & 5340 & - & 5330 & $5350:$ & 5370 & 5340: & 5290: \\
\hline $\mathrm{F}$ & Fe III? (blend) & 5420 & 5610 & $5600:$ & $5580:$ & $5580:$ & $5600:$ & 5570 & - & 5550 & 5540 & 5560 & $5560:$ & 5520: \\
\hline G & Co III? & - & - & 5850: & 5840 & $5850:$ & 5820: & $5890:$ & - & 5910 & 5920 & 5920 & 5930 & 5920 \\
\hline $\mathrm{H}$ & Co III? & - & - & $6060:$ & 6040: & $6060:$ & 6060: & 6070: & - & 6070 & 6150 & 6170: & $6200:$ & 6220: \\
\hline I & Fe II? & - & - & 6550 & $6550:$ & $6540:$ & 6520 : & 6530 & 6530 & 6530 & 6520 & 6520 & $6520:$ & $6520:$ \\
\hline $\mathrm{J}$ & Fe II (blend) & - & - & - & - & - & - & 7270 : & $7280:$ & $7280:$ & $7280:$ & $7260:$ & $7230:$ & $7240:$ \\
\hline $\mathrm{K}$ & $\mathrm{OI}$ & - & - & - & $7750:$ & - & - & - & 7750 & $7740:$ & - & - & - & - \\
\hline $\mathrm{L}$ & Ca II (blend) & - & - & - & 8630 & - & - & - & 8680 & 8680: & - & - & - & - \\
\hline
\end{tabular}

${ }^{a}$ Phase of observation in days past assumed V maximum (JD 2448605.3)

(Table 5). The evolution of these two features might indicate the changing ionization states of $\mathrm{Fe}$ and the cooling of the nebula. An intriguing feature is the absorption at 5700 $\AA$ (j) which does not shift and is very narrow (FWHM $\approx 2500 \mathrm{~km} \mathrm{~s}^{-1}$ ). For comparison the width of the $\mathrm{Ca}$ II absorption at the same epoch (45 days) is $7000 \mathrm{~km} \mathrm{~s}^{-1}$. The origin of the $5700 \AA$ line is unclear, but it is remarkable that the emission line at $5900 \AA(\mathrm{G})$, which is clearly visible by day 45 , is likewise very narrow suggesting small velocities in the ejecta. A possible identification of either feature would be Na I (see also Filippenko et al. 1992b). By day 83 the absorption $\mathrm{j}$ has evolved into a blend, as the sharp blue edge prevails, but the red edge is eroded away by another feature. The spectral changes between 83 and 111 days are minor. The emission lines at this stage are well-defined and most likely identified with forbidden lines of $\mathrm{Fe}$ and $\mathrm{Co}$.

\section{DISCUSSION}

The red color of SN $1991 \mathrm{bg}$ at maximum contrasts with the observed colors of other SNe Ia. Figure 5 shows a histogram of the observed $(\boldsymbol{B}-\boldsymbol{V})$ color at maximum for all $\mathrm{SNe}$ Ia and SNe I as determined by Leibundgut et al. (1991a) supplemented with the measurements of SN 1986G (Phillips et al. 1987), SN 1989B (Barbon et al. 1990; Wells et al. 1992), SN 1990N (Leibundgut et al. 1991b), SN 1991T (Phillips et al. 1992), and SN 1991bg. Interpretation of this diagram is complicated by several effects. Observational errors increase the overall scatter and widen the distribution in a symmetric way. This is probably the cause for the two bluest supernovae. The sharp increase at about $(B-V)_{\max } \approx-0.25$ is a strong argument for a blue limit to the intrinsic colors of SNe Ia. As discussed by van den Bergh \& Pierce (1992), most of the galaxies with such blue supernovae are ellipticals and the spread in color is much larger for SNe Ia in spiral galaxies. In fact, except for SN 1991bg, all supernovae in ellipticals are confined to very blue colors, but some supernovae in $\mathrm{S}_{\text {pec }}$ galaxies have $(B-V)_{\max } \geqslant 0.0$.
Figure 5 shows that only SN $1986 \mathrm{G}$ exhibited a larger $B-V$ at maximum than SN 1991bg. The red color of SN $1986 \mathrm{G}$ has been attributed to extinction in the prominent dust lane of Cen A. This conclusion is supported by observations of strong interstellar lines of $\mathrm{Ca}$ II and $\mathrm{Na} \mathrm{I}$ in the spectrum of SN 1986G (Phillips et al. 1987; Rich 1987; di Serego Alighieri \& Ponz 1987; Christiani et al. 1992). If we were to assume that this is also the explanation for the red color of $\mathrm{SN} 1991 \mathrm{bg}$, then a color excess of $\mathrm{E}(B-V)$ $\approx 0.7$ would be derived (provided the intrinsic color at maximum is 0.0 ) which would imply an extinction of $A_{B}$

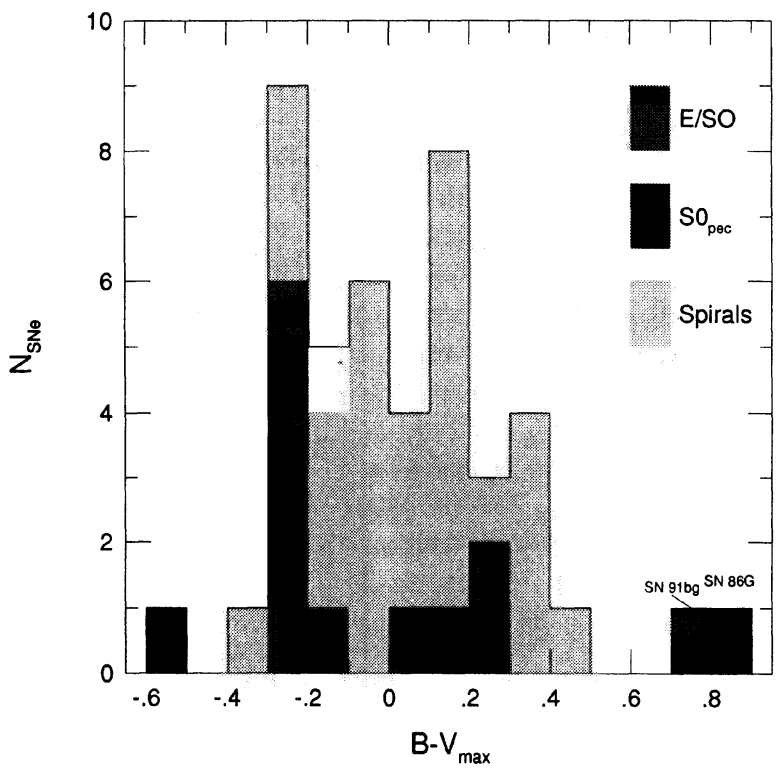

FIG. 5. Histogram of $B-V$ colors observed at maximum for $\mathrm{SNe}$ Ia. The data have been taken from Leibundgut et al. (1991a) and are corrected for Galactic absorption only. Supernovae in elliptical galaxies are the dark shaded area, light shaded areas denote SNe Ia in spiral galaxies, and the rest occurred in $\mathrm{SO}_{\text {pec }}$. Note the sharp turn-on near $B-V_{\max }=-0.3$. SN 1991 bg clearly lies outside the main distribution. The blank space in the histogram is for the parent galaxy of SN 1973B for which no galaxy type was available. 
$\approx 2^{\mathrm{m}} 8$ for a conventional reddening law. Interestingly, this is close to the brightness difference observed between SN $1991 \mathrm{bg}$ and SN 1957B or other SNe Ia in Virgo. There are, however, several arguments against this hypothesis. The color of SN $1991 \mathrm{bg}$ at late times ( $t>35$ days) is as blue or even bluer than the template (Fig. 2) and much bluer than for SN 1986G (Fig. 3). No interstellar absorption lines are detectable in the spectra of SN $1991 \mathrm{bg}$, and no evidence for dust at the supernova position can be extracted from very deep direct images of NGC 4374. From inspection of the deep frames the local absorption in $B$ can be estimated to be less than $0 \mathrm{~m} 1$ at the position of the supernova assuming a conventional absorption law. Another possibility is that condensation of matter in the supernova ejecta itself could have caused the reddening. Dust was observed to condense in the ejecta of SN 1987A around 600 days after outburst (Lucy et al. 1991) without noticeable narrow absorption lines appearing in the spectra (e.g., Phillips et al. 1990). The evidence from the color and light curves, however, seem to exclude such an explanation for SN 1991bg.

It has been suggested that $\mathrm{SNe}$ Ia are good standard candles (e.g., Miller \& Branch 1990, 1992; Leibundgut \& Tammann 1990; Tammann \& Leibundgut 1990), despite the strong spectral deviations observed in a few cases (Branch et al. 1988; Filippenko et al. 1992a; RuizLapuente et al. 1992; Phillips et al. 1992). A few events, however, have displayed distinct light curves, foremost SN 1986G (Phillips et al. 1987; Frogel et al. 1987) and possibly SN 1939B (e.g., Leibundgut et al. 1991a). Most SNe Ia with well-observed light curves, however, appear to define a narrow zone in a redshift versus apparent magnitude diagram (Leibundgut \& Pinto 1992; see, however, van den Bergh \& Pierce 1992) and only a small scatter of the absolute $B$ magnitude at maximum is observed (Miller \& Branch 1992; Della Valle \& Panagia 1992). SN 1991bg does not fit into this picture.

The low peak luminosity of SN 1991bg is most readily apparent when contrasted with SN 1957B which occurred in the same galaxy (Bertola 1964; Goetz 1957; Romano 1957; Li Tzin 1957; Greenstein \& Minkowski 1973), and reached a maximum magnitude of 12.2 in the photographic bandpass (Leibundgut et al. 1991a). The spectrum of SN 1957 B at $\sim 24$ days published by Greenstein \& Minkowski (1973) with comparable epoch spectra of SN 1989B (Wells et al. 1992) shows unambiguously that SN 1957B was a type Ia supernova. The magnitude difference between SN 1957B and SN 1991bg amounts to about $2.5 \mathrm{mag}$ in $B$ (assuming that the $B$ maximum of SN $1991 \mathrm{bg}$ was around 14.7).

NGC 4374 (M 84) is an E1 galaxy with small $\mathrm{H}$ I content (only upper limits have been found to date; van Gorkom et al. 1989) and no detection of $\mathrm{CO}$ emission (Rupen 1992). A radio jet extends from the center about 1 ' in the north-south direction (Laing \& Bridle 1987; Gregorini et al. 1989). The galaxy has been detected in X-rays (Forman et al. 1985; Canizares et al. 1987) and at all wavelengths by IRAS (Knapp et al. 1989). A dust lane in the core extending in the E-W direction (Veron-Cetty \& Veron 1988; Hansen et al. 1985; Gallagher 1986) coincides

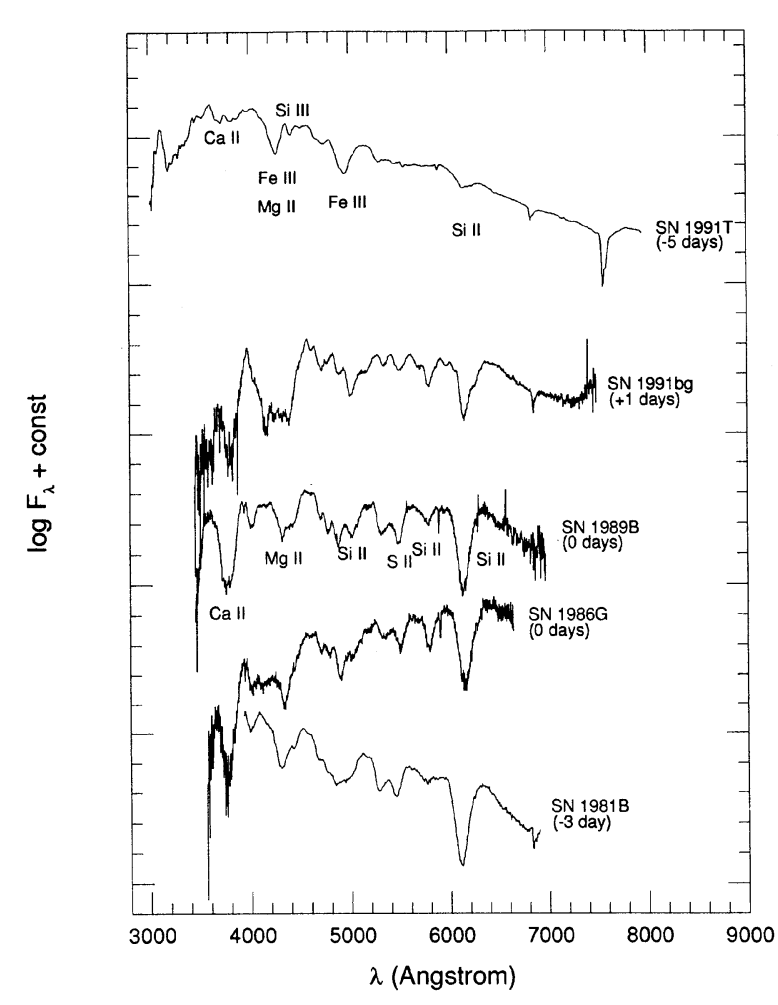

FIG. 6. Spectral comparison of SNe Ia near maximum. The spectra are shifted by arbitrary amounts along the ordinate and have been corrected for the observed galaxy redshifts. The epochs are days past the $V$ maximum of each supernova. The sources for the spectra are Phillips et al. (1992; SN 1991T), Wells et al. (1992; SN 1989B), Phillips et al. (1987; SN 1986G), and Branch et al. (1983; SN 1981B).

with $\mathrm{H} \alpha$ emission (Baum et al. 1988; Trinchieri \& di Serego Alighieri 1990). NGC 4374 has a radial velocity of $930 \mathrm{~km} \mathrm{~s}^{-1}$ (Huchtmeier \& Richter 1989) and is listed as a member of the Virgo cluster in the VCC (Binggeli et al. 1985). Distances for NGC 4374 have been obtained with the planetary nebula luminosity. functions (Jacoby et al. 1990) and surface brightness fluctuations (Tonry et al. 1990) and both place the galaxy slightly behind the cluster center, but by less than 10\%. Samples of SNe Ia in Virgo yield an average of $m_{B}=12.0 \pm 0.2$ (Leibundgut \& Tammann 1990; Capaccioli et al. 1990). The maximum of SN 1957B is consistent with this value, but SN 1991bg was more than 2 mag fainter in B. Thus, $S N 1991$ b clearly did not reach a maximum luminosity comparable to other $S N e$ Ia. This appears to be the first unambiguous example of an intrinsically dim type Ia supernova.

It is instructive to compare the optical spectra of SN $1991 \mathrm{bg}$ with observations at similar epochs of other $\mathrm{SNe}$ Ia. At maximum light the $\mathrm{SNe}$ Ia spectrum is produced by the outermost material in the ejecta and is composed mainly of lines of intermediate mass elements (Branch et al. 1985; Harkness 1991a,b; Jeffery et al. 1992). Figure 6 displays the spectrum of SN $1991 \mathrm{bg}$ along with four other SNe Ia for which an observation near maximum light was available. All spectra have been corrected for the observed 
galaxy redshifts tabulated by Huchtmeier \& Richter (1990). The line identifications are taken from Jeffery et al. (1992) for SN 1991T and Harkness (1991b) for SN 1989B. SN 1986G and SN 1989B were reddened considerably by interstellar material as supported by the strengths of the narrow $\mathrm{Na} \mathrm{D}$ absorptions in their spectra. The spectrum of SN 1991T is from a significantly earlier phase than the other spectra and the continuum slope is still quite blue. With the exception of SN 1991T, all spectra are similar, but slight differences in line strengths and velocities are clearly present. The characteristic Si II absorption at $6150 \AA$ is visible in all spectra, but with considerably different strengths (see also Filippenko et al. 1992a; RuizLapuente et al. 1992; Phillips et al. 1987, 1992). As in SN $1991 \mathrm{bg}$ this feature is also blended on the red side in SN 1986G. A search for a similar extension of the Si II multiplet at $5900 \AA$ in the spectrum of this $\mathrm{SN}$ is hampered by the strong interstellar absorption due to $\mathrm{Na}$ I. Another feature which varies significantly among the different supernovae is the Si II line at $5800 \AA$ (Phillips \& Hamuy 1991). The $\mathrm{Ca}$ II $\mathrm{H} \& \mathrm{~K}$ lines are observed in emission in $\mathrm{SN}$ 1986G, SN 1989B, and SN 1991bg. The absorption component is also easily visible in all three SNe. Strong differences are observed in the region from $\sim 4000$ to $\sim 4200 \AA$. Si II absorption (from the $4130 \AA$ multiplet) is present at $\sim 4000 \AA$ in the spectra of SN 1981B, SN 1989B, and more weakly in SN 1986G. No trace of this line is visible in SN 1991T and it is possibly filled in by the Ca II H \& K emission in SN 1991bg. Note also that the sharpness of the individual features is different among the individual $\mathrm{SNe}$.

Two weeks past maximum SN 1991bg enters into a transitional period when the photosphere recedes to the center and a nebular spectrum begins to emerge. At this stage spectra are composites of newly forming emission features and slowly disappearing absorptions. Spectra at this epoch are shown for five SNe Ia in Fig. 7. In contrast to the situation near maximum light, the supernovae are much more homogeneous at this epoch (Filippenko et al. 1992a; Phillips et al. 1992). SN 1991T, which was clearly discrepant two weeks earlier, displays the same bumps and wiggles as SN 1990N, SN 1989B, and SN 1981B. The main difference at this phase is the strength of the Si II line at $6150 \AA$. Slight differences in the $4000-5000 \AA$ region are evident in these SNe Ia, but all features are present. The spectrum of SN $1991 \mathrm{bg}$, however, still clearly deviates from the other SNe. Although most of the lines have counterparts in the spectra of other SNe Ia their relative line strengths are different. The $\mathrm{Ca}$ II lines are still very strong and the emission of the infrared triplet shows structure not observed in SN 1990N. The absorption at $4400 \AA$ ( Mg II and $\mathrm{Fe}$ III), which is seen in all other SNe Ia, is conspicuously absent. On the other hand a strong emission identified with [Fe III] (Axelrod 1980a) has formed at $5580 \AA$. This emission has alternatively been identified with the [O I] $\left(\lambda_{0}=5577 \AA\right.$ ) line (Filippenko et al. 1992b). However, the strength of this line and the absence of the [O I] doublet at $6300 \AA$ would imply very high densities of the order of $10^{11} \mathrm{O}^{0}$ atoms $\mathrm{cm}^{-3}$ and low temperatures $\left(T_{e} \leqslant 2000 \mathrm{~K}\right)$ for reasonable electron densities $\left(n_{e} / n_{\mathrm{O}}\right.$

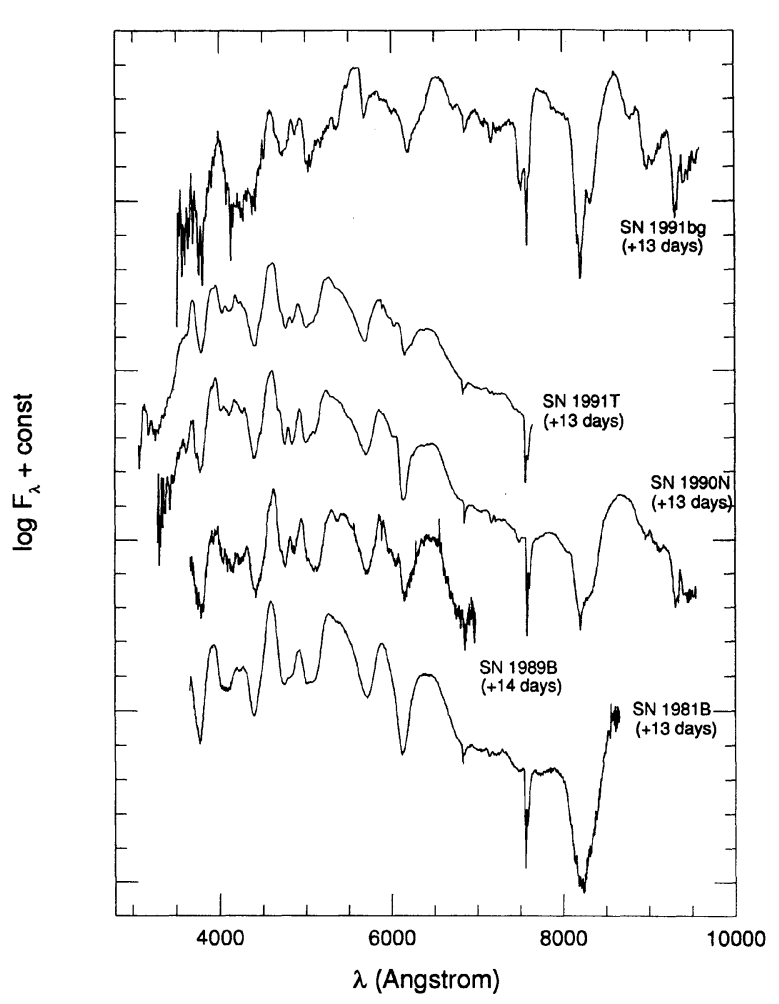

FIG. 7. Same as Fig. 6 for SNe Ia two weeks past maximum. The references are the same as in Fig. 6 with the spectrum of SN 1990N observed on 23.98 July 1990 at CTIO.

$=1)$ and ionization $\left(n_{\mathrm{O} \text { II }} / n_{\mathrm{O}}=10^{-2}\right.$; Leibundgut et al. $1991 \mathrm{c})$. The $5577 /(6300+6363)$ ratio would then decrease with the aging supernova. It would imply that we see a substantial fraction of unburnt oxygen in very dense states outside the ejecta. While not completely excluded by the observations, we think that this interpretation is flawed due to the high densities of neutral oxygen required. The Si II ( $6150 \AA)$ absorption is blended stronger with the unidentified absorption on the red side. Hints of this absorption are also visible in the spectrum of SN 1991T. The emission part of the O I $\left(\lambda_{0}=7774 \AA\right)$ scattering line appears stronger than in SN $1990 \mathrm{~N}$ and SN 1981B. The strength of the $\mathrm{Ca}$ II and $\mathrm{O}$ I emissions indicate the presence of these elements in abundance in the ejecta. The emergence of the $\mathrm{Fe}$ emission at $5600 \AA$ so early suggests a rapid spectroscopic evolution of SN 1991bg as seen in the light curves.

At epochs later than a month after maximum, the spectra of typical SNe Ia are dominated by emission due to ions of $\mathrm{Co}$ and $\mathrm{Fe}$ which were originally deep within the explosion and which are a direct consequence of the basic physical event of a SN Ia-nuclear burning to iron peak elements. As illustrated in Fig. 8, the agreement at these epochs is striking. Differences among the spectra are now dominated by signal-to-noise ratios and resolution. The exception to this rule is again SN 1991bg. Although the general resemblance to a typical type Ia supernova is now much closer, there remain obvious differences. First, the 


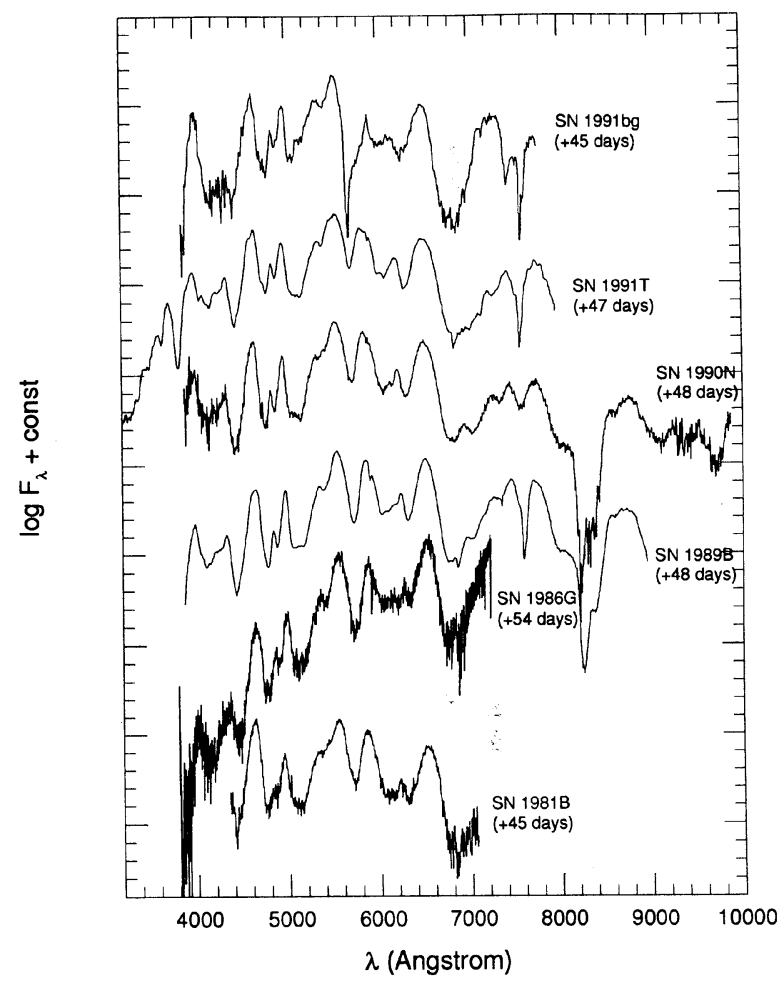

FIG. 8. Same as Fig. 6 for SNe Ia about seven weeks past maximum. Note the homogeneity of all spectra presented except the one of SN 1991 bg.

narrow absorption line at $5700 \AA$ (j) and the adjacent emission (G), normally attributed to [Co III], are distinct from all other SNe. An alternate identification for these lines could be the Na I D lines (Filippenko et al. 1992b). An apparent absorption is also observed at $7430 \AA$ which is not seen in any of the other supernovae. Finally, the Ca II emission is still strong in the spectrum of SN $1991 \mathrm{bg}$.

The expansion velocity as inferred from the observed minimum of the Si II $\left(\lambda_{0}=6355 \AA\right)$ line is a diagnostic of SN Ia explosions (Branch 1987; Branch et al. 1988; Phillips et al. 1992). Figure 9 presents the data for wellobserved SNe Ia. All data have been corrected for the observed redshifts of the parent galaxies and a rise time between explosion and maximum of 20 days has been assumed. Near maximum the majority of supernovae have expansion velocities between 10000 and $13000 \mathrm{~km} \mathrm{~s}^{-1}$ (Branch et al. 1988). SN 1991bg is no exception to this, although its velocity is slightly lower than for most other well-observed SNe Ia. After 10 days SN 1991T and SN $1990 \mathrm{~N}$ showed little change in the observed velocities. This was interpreted as a strong confinement of the Si layer in the explosion (Phillips et al. 1992). SN 1991bg, like SN 1981B, SN 1986G, and SN 1989B, did show decreasing velocities with time. The slopes are comparable for these four SNe, but SN 1991bg has the steepest slope and extends to the lowest velocities at later phases. The rise time of SN 1991bg is not known. We simply assumed that it would be the same as for other SNe Ia, but this might not

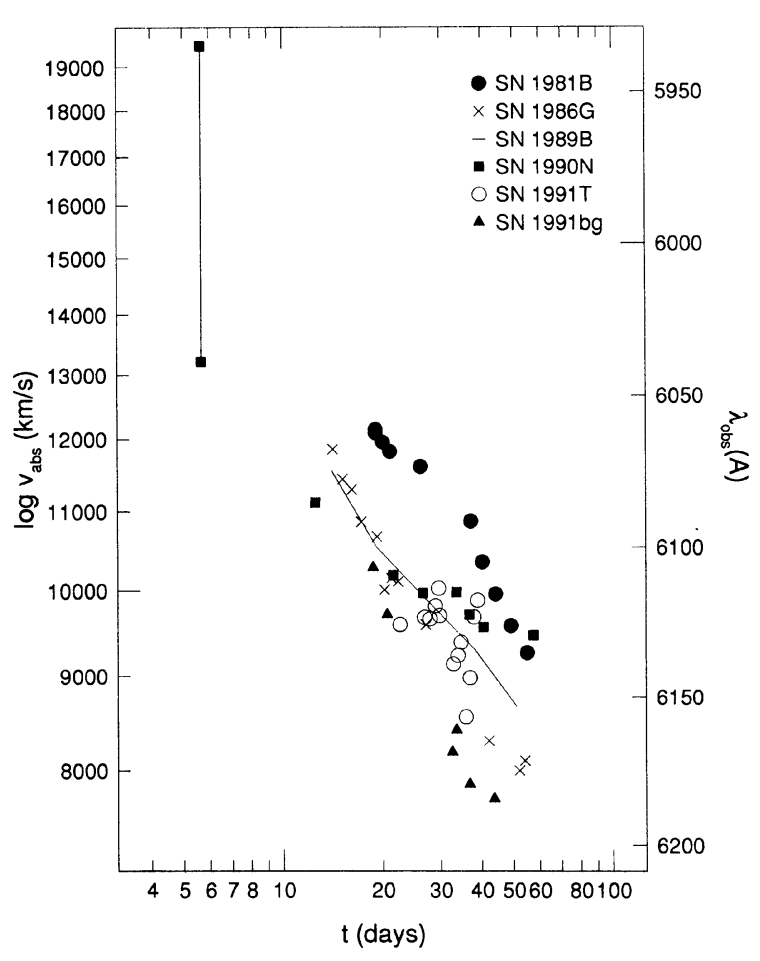

FIG. 9. Expansion velocities inferred from the minimum of the Si II $(6355 \AA$ ) line vs time since explosion. A rise time between explosion and maximum of 20 days has been assumed for all supernovae. The data for the other well-observed SNe Ia (cf. Phillips et al. 1992; Christiani et al. 1992) have been included for comparison.

be a valid procedure in light of the various peculiarities observed in this event. In fact, there might be an indication of a faster rise of SN 1991g in the premaximum observations of Kosai et al. (1991). Shortening the rise time of SN 1991 bg would result in a shallower slope of the velocity evolution.

Many of the peculiar features of SN $1991 \mathrm{bg}$ can be accounted for by a smaller mass in the ejecta. The rapid decline in the light curve and the early color evolution in combination with the almost normal expansion velocities are signs of a smaller column depth in the ejecta than in ordinary $\mathrm{SNe} \mathrm{Ia}$. This also suggests that the low luminosity of SN $1991 \mathrm{bg}$ is due to less efficient trapping of $\gamma$ rays from radioactive ${ }^{56} \mathrm{Ni}$ and ${ }^{56} \mathrm{Co}$ and a shorter photon diffusion time near maximum light. The early transition from a spectrum with scattering lines on a photosphere to emission lines is also consistent with this view. Based on the simple arguments presented in Jeffery et al. (1992) we estimate an ejecta mass of $\sim 0.5-0.8 \mathscr{M}_{\odot}$ for SN $1991 \mathrm{bg}$ assuming that the density structure and composition (mainly Fe-peak elements) are similar to normal SNe Ia.

The nearly normal velocities in combination with the smaller total mass would imply a less energetic explosion. The smaller mass of synthesized material would also lower the peak luminosity. Yet the spectroscopic similarities of the interior, as indicated in Fig. 8, demand that the nuclear burning proceed to the usual composition in the inner 
zones. To some extent, the observed homogeneity of $\mathrm{SNe}$ Ia has seemed more plausible because of the very welldefined physical event of exploding a white dwarf at the Chandrasekhar limit. Perhaps the peculiarity of SN 1991bg stems from a variant of an explosion at a somewhat lower mass.

\section{CONCLUSIONS}

SN 1991bg is an unusual type Ia supernova in several ways - the quick fading after an underluminous maximum, the red $B-V$ color at maximum and its subsequent evolution, the relative strengths of the lines in the spectra and their evolution. Most unusual is the brief duration of the photospheric phase which ended just two weeks after maximum. What SN 1991bg does have in common with other $\mathrm{SNe}$ Ia are the strong Si II and Ca II lines near maximum light. Indeed, the classification of SN 1991bg as a type Ia seems reasonable since it has the characteristic Si II $6150 \AA$ line in the maximum light spectra, the usual $\mathrm{Fe}$ emission lines and the blue $B-V$ color at late phases ( $t \geqslant 40$ days). Furthermore, the slopes in the light and color curves are almost identical with the templates at late times. This implies that SN 1991bg represents a variation of the general SN Ia event rather than a fundamentally different object. Thus, we think that a further subclassification for this supernova is not warranted.

Our discussion has assumed that the red color of SN $1991 \mathrm{bg}$ is an intrinsic property and not due to attenuation by dust. Reddening from the Galaxy can be excluded based on the observations of SN 1957B in the same galaxy and the lack of narrow interstellar absorption lines in the spectra. The absence of interstellar lines at the redshift of NGC 4374 also argues for little absorption in the parent galaxy as long as the gas-to-dust ratio is not very small. Moreover, no obvious dust features have been observed at the position of SN $1991 \mathrm{bg}$ in NGC 4374. The most convincing argument, however, is the relative blue color at late times, when SN 1991bg exhibits similar colors to other SNe Ia.

The spectral evolution of SN 1991bg is unique but not unrecognizable. The photospheric phase resembles other $\mathrm{SNe} \mathrm{Ia}$, but the early changes and the development of emission features clearly distinguish this supernova from other type Ia events. This calls the dating of SNe Ia based on single spectra alone into question. The peculiarities in the maximum light spectra of SN $1991 \mathrm{bg}$ consist mostly of changed line ratios compared to normal SNe Ia. Thus, the outer layers of SN $1991 \mathrm{bg}$ appear closely related to those of other SNe Ia implying that the end stages of the burning were comparable. The short duration of the photospheric phase is taken as a sign of a smaller ejecta mass in SN $1991 \mathrm{bg}$. The nebular phase spectra are dominated by broad emission lines as is common for SNe Ia. Real differences from normal SNe Ia are, however, still present. Most notable is a narrow absorption trough accompanied by a narrow emission line in the region between 5700 and $6000 \AA$. A possible explanation for this feature could be blending of a resonance line with a pure emission, the latter possibly from [Co III] lines observed in normal SNe Ia (Axelrod 1980a,b).

Except for SN 1991bg all SNe Ia with observations at late epochs ( $t \geqslant 40$ days) display a high degree of uniformity providing evidence that the stellar interiors resemble one another. This is in accord with what is expected from a thermonuclear explosion of a white dwarf (Nomoto et al. 1984; Woosley et al. 1986; Axelrod 1980b). Even supernovae with peculiarities at earlier phases exhibit very similar spectra at the nebular stage. An excellent example of this is SN 1991T which had a very peculiar premaximum spectrum (Filippenko et al. 1992a; Ruiz-Lapuente et al. 1992, Phillips et al. 1992). Even SN 1986G, which had fast decaying $B$ and $V$ light curves after maximum (Phillips $e t$ al. 1987) and peculiar infrared light curves (Frogel et al. 1987), was spectroscopically indistinguishable from other $\mathrm{SNe}$ Ia at late epochs. Hence, the unusual nature of SN $1991 \mathrm{bg}$ is even more striking.

Filippenko et al. (1992b) discuss several possible explosion scenarios which could explain the observations of SN $1991 \mathrm{bg}$. The facts presented here support their main conclusion that the ejecta mass is lower than for normal $\mathrm{SNe}$ Ia. If it is possible to explode a white dwarf at a lower mass or to lock up mass in a compact remnant, an event resembling SN 1991bg might well result.

Finally, this peculiar event complicates the question whether SNe Ia make good standard candles. We think that SN 1991bg is peculiar in so many aspects that it clearly is separable as a distinct event within the class of SNe Ia. Even if the apparent brightness of SN 1991bg were comparable to SN 1957B and other SNe Ia within the Virgo cluster, the photometric and spectroscopic evolution of SN 1991bg is sufficiently different that it would have been recognized as unusual. The faintness of SN 1991bg, nevertheless, demands that future use of SNe Ia as distance indicators has to be tied to an identification scheme that can sort out "suitable" objects for distance determination. One such criterion could be the brightness evolution as measured by the light and color curves and the colors at maximum. Most SNe Ia do follow the template light curves fairly well (to within $0^{\mathrm{m}} 2$ ) and the color at maximum exhibits only a small scatter even when no correction is made for reddening in the parent galaxies (Leibundgut 1991). Peculiar SNe Ia, such as SN 1986G, SN 1991bg, and possibly SN 1939B issue their own warnings that they should be excluded for distance purposes. The danger is that distant supernovae with these characteristics may be included in estimates for the distance scale when the data are incomplete.

We are grateful to P. Pinto, F.-K. Thielemann, and D. Jeffery for many helpful discussions on the nature of type Ia supernovae, J. Tonry for kindly making his deep images of NGC 4374 available to us, and A. Filippenko for sending us a draft of their paper on SN 1991bg. The research at the CfA is supported by the NSF Grant No. AST 89-05529 and the NASA Grant No. NAG 5-841 to R.P.K. 


\section{REFERENCES}

Axelrod, T. S. 1980a, Ph.D. thesis, University of California, Santa Cruz Axelrod, T. S. 1980b, Type I Supernovae, edited by J. C. Wheeler (University of Texas at Austin, Austin), p. 80

Baade, W. 1964, as reported by R. Minkowski, ARA\&A, 2, 247

Barbon, R., Benetti, S., Cappellaro, E., Rosino, L., \& Turatto, M. 1990, A\&A, 237, 79

Barbon, R., Ciatti, F., \& Rosino, L. 1973, A\&A, 25, 65

Barbon, R., Iijima, T., \& Rosino, L. 1989, A\&A, 220, 83

Baum, S. A., Heckman, T., Bridle, A., van Breugel, W., \& Miley, G. 1988, ApJS, 74, 389

Benetti, S., Cappellaro, E., \& Turatto, M. 1991, IAU Circ. No. 5405 Bertola, F. 1964, AJ, 69, 236

Bertola, F., Mammano, A., \& Perinotto, M. 1965, Contr. Asiago No. 174 Binggeli, B., Sandage, A., \& Tammann, G. A. 1985, AJ, 90, 1681

Blair, W. P., \& Panagia, N. 1987, Exploring the Universe with the IUE

Satellite, edited by Y. Kondo et al. (Kluwer, Dordrecht), p. 549

Boisseau, J. R., \& Wheeler, J. C. 1991, AJ, 101, 1281

Branch, D. 1987, ApJ, 316, L81

Branch, D., Doggett, J. B., Nomoto, K., \& Thielemann, F.-K. 1985, ApJ, 294, 619

Branch, D., Drucker, W., \& Jeffery, D. J. 1988, ApJ, 330, L117

Branch, D., Lacy, C. H., McCall, M. L., Sutherland, P. G., Uomoto, A., Wheeler, J. C., \& Wills, B. J. 1983, ApJ, 270, 123

Burstein, D., Davies, R. L., Dressler, A., Faber, S. M., Stone, R. P. S.,

Lynden-Bell, D., Terlevich, R. J., \& Wegner, G. 1987, ApJS, 64, 601

Campbell, L. 1939, Harvard Repr. 190, 31

Canizares, C. R., Fabbiano, G., \& Trinchieri, G. 1987, ApJ, 312, 503

Capaccioli, M., Cappellaro, E., Della Valle, M., D’Onofrio, M., Rosino,

L., \& Turatto, M. 1990, ApJ, 350, 110

Christiani, S., et al. 1992, A\&A, 259, 63

Della Valle, M., Danziger, J., \& Gouiffes, C. 1991, IAU Circ. No. 5408

Della Valle, M., \& Panagia, N. 1992, AJ, 104, 969

di Seregho Alighieri, S., \& Ponz, D. 1987, ESO Workshop on Supernova

1987A, edited by I. J. Danziger (ESO, Garching), p. 545

Filippenko, A. V. 1989, PASP, 101, 588

Filippenko, A. V., Dey, A., van Breugel, W. J. M., \& Deustua, S. 1991, IAU Circ. 5403

Filippenko, A. V., et al. 1992a, ApJ, 384, L15

Filippenko, A. V., et al. 1992b, AJ, 104, 1543

Forman, W., Jones, C., \& Tucker, W. 1985, ApJ, 293, 102

Frogel, J. A., Gregory, B., Kawara, K., Laney, D., Phillips, M. M.

Terndrup, D., Vrba, F., \& Whitford, A. E. 1987, ApJ, 315, L129

Gallagher, J. 1986, PASP, 98, 81

Goetz, W. 1957, ANac, 284, 141

Graham, J. A. 1982, PASP, 94, 244

Greenstein, J. L., \& Minkowski, R. 1973, ApJ, 182, 225

Gregorini, L., Messina, A., \& Vettolani, G. 1989, A\&AS, 80, 239

Hamuy, M., Phillips, M. M., Maza, J., Wischnjewsky, M., Uomoto, A., Landolt, A. U., \& Kathwani, R. 1991, AJ, 102, 208

Hamuy, M., Maza, J., Antezana, R., Wells, L., Smith, R. C., Suntzeff, N., \& Phillips, M. M. 1992, IAU Circ. No. 5468

Hansen, L., Nørgaard-Nielsen, H. U., \& Jørgensen, H. E. 1985, A\&A, 149,442

Harkness, R. P. 1991a, Supernovae, edited by S. E. Woosley (Springer, New York), p. 454

Harkness, R. P. 1991b, SN 1987A and Other Supernovae, edited by I. J. Danziger and K. Kjär (ESO, Garching), p. 447

Harkness, R. P., \& Wheeler, J. C. 1990, Supernovae, edited by A. G. Petschek (Springer, New York), p. 1

Hoffleit, D. 1939, Harvard Coll. Ann. Card 487

Huchtmeier, W. K., \& Richter, O.-G. 1989, A General Catalog of H Observations of Galaxies (Springer, New York)

Jacoby, G. H., Ciardullo, R., \& Ford, H. C. 1990, ApJ, 356, 332
Jeffery, D. J., Leibundgut, B., Kirshner, R. P., Benetti, S., Branch, D., \& Sonneborn, G. 1992, ApJ, 397, 304

Kimeridze, G. N., \& Tsvetkov, D. Y. 1986, Astrofizika, 25, 279

Kirshner, R. P., Peters, J., \& Leibundgut. B. 1991, IAU Circ. No. 5403

Kirshner, R. P., Oke, J. B., Penston, M. V., \& Searle, L. 1973, ApJ, 185, 303

Kirshner, R. P., \& Oke, J. B. 1975, ApJ, 200, 574

Knapp, G. R., Guhathakurta, P., Kim, D.-W., \& Jura, M. 1989, ApJS, 70,329

Kosai, H., Kushida, R., Kushida, Y., \& Kato, T. 1991, IAU Circ. No. 5400

Laing, R. A., \& Bridle, A. H. 1987, MNRAS, 228, 557

Landolt, A. U. 1983, AJ, 88, 439

Leibundgut, B. 1988, Ph.D. thesis, University of Basel

Leibundgut, B. 1991, Supernovae, edited by S. E. Woosley (Springer, New York), p. 751

Leibundgut, B., Tammann, G. A., Cadonau, R., \& Cerrito, D. 1991a, A\&AS, 89, 537

Leibundgut, B., Kirshner, R. P., Filippenko, A. V., Shields, J. C., Foltz, C. B., Phillips, M. M., Sonneborn, F. 1991b, ApJ, 371, L23

Leibundgut, B., Kirshner, R. P., Pinto, P. A., Rupen, M. P., Smith, R. C., Gunn, J. E., \& Schneider, D. P. 1991c, ApJ, 372, 531

Leibundgut, B., \& Pinto, P. A. 1992, ApJ (in press)

Leibundgut, B., \& Tammann, G. A. 1990, A\&A, 230, 81

Li Tzin 1957, AcASn, 5, 321

Lucy, L. B., Danziger, I. J., Gouiffes C., \& Bouchet, P. 1991, Supernovae, edited by S. E. Woosley (Springer, New York), p. 82

Miller, D. L., \& Branch, D. 1990, AJ, 100, 530

Miller, D. L., \& Branch, D. 1992, AJ, 102, 379

Minkowski, R. 1941, PASP, 53, 224

Minkowski, R. 1964, ARA\&A, 2, 247

Nomoto, K., Thielemann, F.-K., \& Yokoi, K. 1984, ApJ, 286, 644

Phillips, M. M., Wells, L. A., Suntzeff, N. B., Hamuy, M., Leibundgut, B., Kirshner, R. P., \& Foltz, C. B. 1992, AJ, 103, 1632

Phillips, M. M., \& Hamuy, M. 1991, IAU Circ. No. 5408

Phillips, M. M., Hamuy, M., Heathcote S. R., Suntzeff, N. B., \& Kirhakos, S. 1990, AJ, 99, 1133

Phillips, M. M., et al. 1987, PASP, 99, 592

Polcaro, V. F., \& Viotti, R. 1991, A\&A, 242, L9

Pskovskii, Y. P. 1967, Sov. Astron., 11, 63

Pskovskii, Y. P. 1971, Sov. Astron., 14, 798

Pskovskii, Y. P. 1984, Sov. Astron., 28, 658

Rich, R. M. 1987, AJ, 94, 651

Romano, G. 1957, Comm. Padova No. 1

Ruiz-Lapuente, M. P. 1992, Ph.D. thesis, University of Barcelona

Ruiz-Lapuente, P., Cappellaro, E., Turatto, M., Gouiffes, C., Danziger, I J., Della Valle, M., \& Lucy, L. B. 1992, ApJ, 387, L33

Rupen, M. 1992, private communication

Shapley, H. 1939, Harvard Coll. Ann. Card 487

Tammann, G. A., \& Leibundgut, B. 1990, A\&A, 236, 9

Tonry, J. L., Ajhar, E. A., \& Luppino, G. A. 1990, AJ, 100, 1416

Trinchieri, G., \& di Serego Alighieri 1990, AJ, 101, 1647

van den Bergh, S., \& Pierce, M. J. 1992, PASP, 104, 408

van Gorkom, J. H., Knapp, G. R., Ekers, R. D., Ekers, D. D., Laing, R

A., \& Polk, K. S. 1989, AJ, 97, 708

Veron-Cetty, M.-P., \& Veron, P. 1988, A\&A, 204, 28

Wells, L. A., et al. 1992, in preparation

Woosley, S. E., Taam, R. E., \& Weaver, T. A. 1986, ApJ, 301, 601

Zwicky, F. 1962, Problems of Extragalactic Research, edited by G. C.

McVittie (MacMillan, New York), p. 347

Zwicky, F. 1965, Stellar Structure, edited by L. H. Aller and D. B. McLaughlin (University of Chicago Press, Chicago), p. 367 


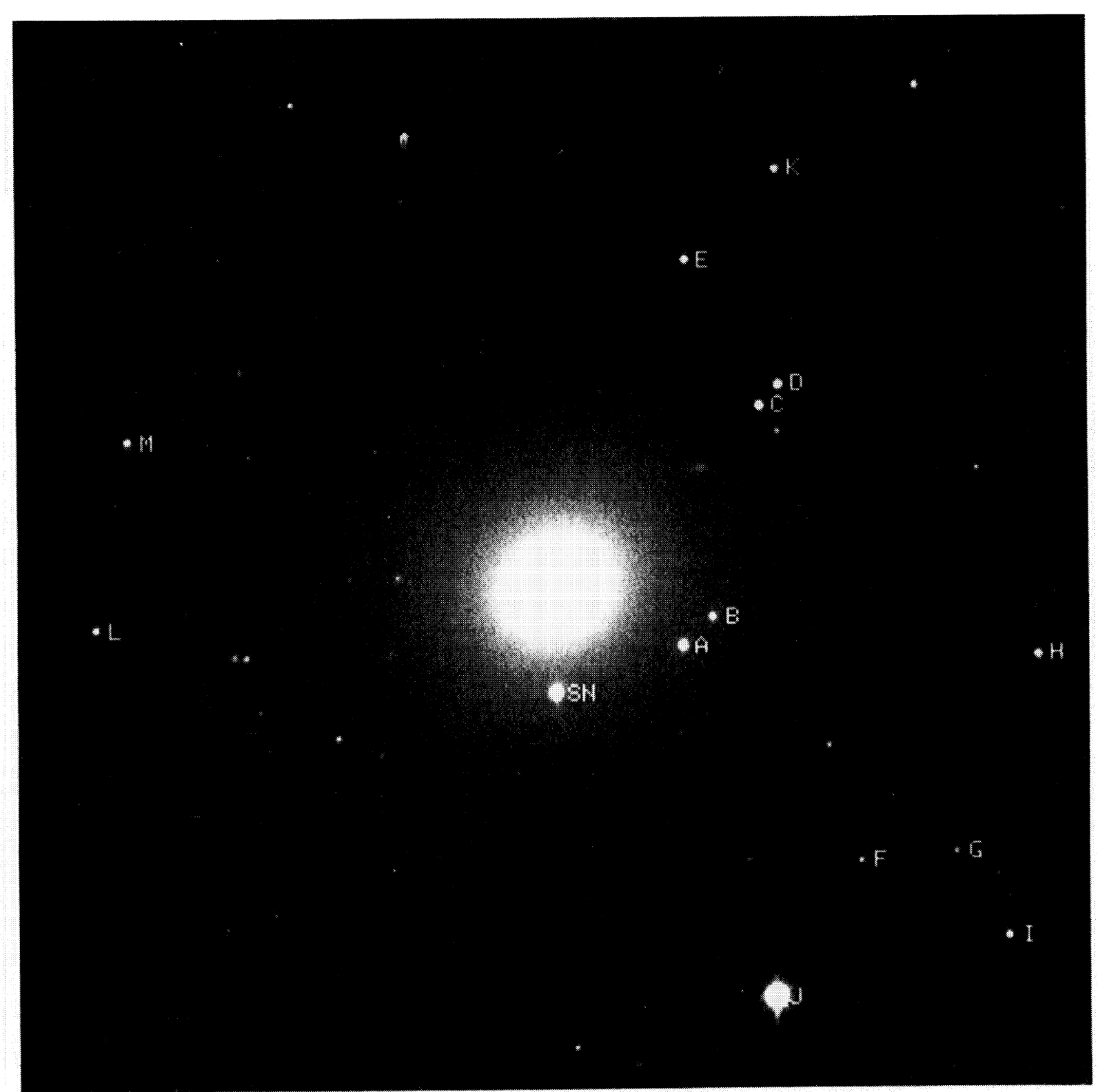

FIG. 1. $B$ filter image of SN $1991 \mathrm{bg}$ in NGC 4374. This frame was observed with the $1.2 \mathrm{~m} \mathrm{SAO}$ telescope at the Whipple Observatory with a Loral $2048^{2} \mathrm{CCD}$ on 15.55 December 1991 (UT). The picture is the sum of two $300 \mathrm{~s}$. exposures. The supernova and the local standard stars are labeled. North is up and east to the left; the field of view is $10^{\prime}$ on a side.

Leibundgut et al. (see page 304) 